Accepted Manuscript

Optimal Regime Switching under Risk Aversion and Uncertainty

Michail Chronopoulos, Sara Lumbreras

PII:

S0377-2217(16)30447-7

DOI:

10.1016/j.ejor.2016.06.027

Reference:

EOR 13779

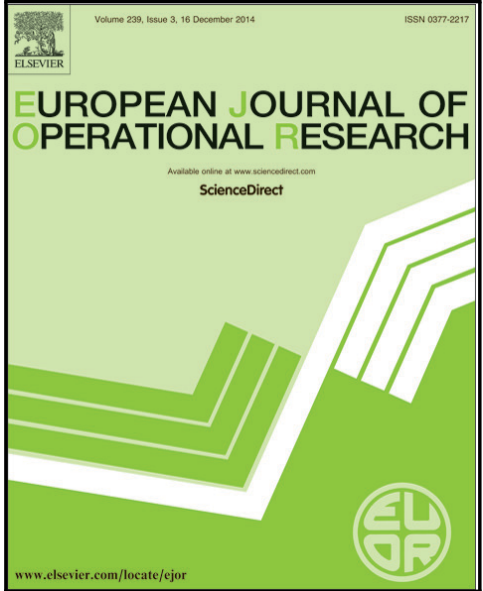

To appear in: $\quad$ European Journal of Operational Research

Received date: 28 December 2015

Revised date: $\quad 20$ May 2016

Accepted date: $\quad 13$ June 2016

Please cite this article as: Michail Chronopoulos, Sara Lumbreras, Optimal Regime Switching under Risk Aversion and Uncertainty, European Journal of Operational Research (2016), doi: 10.1016/j.ejor.2016.06.027

This is a PDF file of an unedited manuscript that has been accepted for publication. As a service to our customers we are providing this early version of the manuscript. The manuscript will undergo copyediting, typesetting, and review of the resulting proof before it is published in its final form. Please note that during the production process errors may be discovered which could affect the content, and all legal disclaimers that apply to the journal pertain. 


\section{Highlights}

- We develop a regime-switching, utility-based framework for sequential investment

- Greater price uncertainty and risk aversion may accelerate regime switching

- Greater risk aversion promotes a compulsive and a laggard regime-switching strategy

- Higher output price or innovation rate mitigates the impact of risk aversion

- A leapfrog/laggard strategy may dominate even when a decisionmaker is risk averse

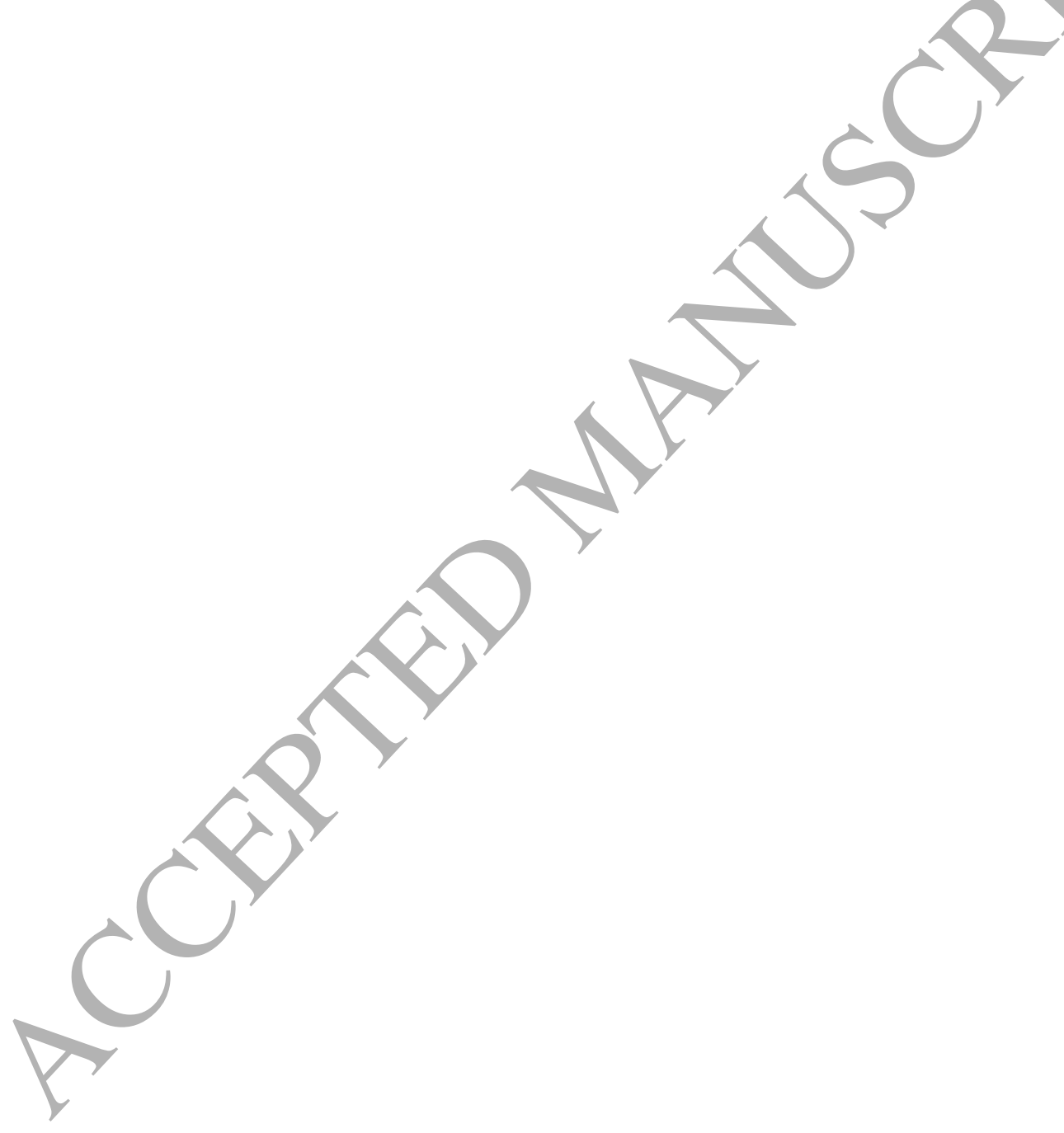




\title{
Optimal Regime Switching under Risk Aversion and Uncertainty
}

\author{
Michail Chronopoulos
}

University of Brighton, School of Computing Engineering and Mathematics, Brighton, UK, Email: Michail.Chronopoulos@nhh.no,Telephone: +44(0)r710808417

Norwegian School of Economics,Department of Business and Management Science Bergen, Norway

Sara Lumbreras

Universidad Pontificia Comillas, Madrid, Spain

\begin{abstract}
Technology adoption is key for corporate strategy, often determining the success or failure of a company as a whole. However, risk aversion often raises the reluctance to make a timely technology switch, particularly when this entails the abandonment of an existing market regime and entry in a new one. Consequently, which strategy is most suitable and the optimal timing of regime switch depends not only on market factors, such as the definition of the market regimes, as well as economic and technological uncertainty, but also on attitudes towards risk. Therefore, we develop a utility-based, regime-switching framework for evaluating different technology-adoption strategies under price and technological uncertainty. We assume that a decisionmaker may invest in each technology that becomes available (compulsive) or delay investment until a new technology arrives and then invest in either the older (laggard) or the newer technology (leapfrog). Our results indicate that, if market regimes are asymmetric, then greater risk aversion and price uncertainty in a new regime may accelerate regime switching. In addition, the feasibility of a laggard strategy decreases (increases) as price uncertainty in an existing (new) regime increases. Finally, although risk aversion typically favours a compulsive and a laggard strategy, a leapfrog strategy may be feasible under risk aversion provided that the output price and the rate of innovation are sufficiently high. Keywords: investment analysis, real options, regime switching, risk aversion, dynamic programming
\end{abstract}




\section{Introduction}

Within an environment of rapid technological innovation and increasing economic uncertainty, reluctance towards technological change may have devastating consequences for the viability of private firms (Hoppe, 2002; Bos et al., 2013). For example, in 1976 Kodak held an impressive market share of $90 \%$ in film photography in the US and owned an extensive portfolio of valuable patents, including digital photography. Yet in 2012 it filed for bankruptcy, displaced by the same technology it had initiated, as it failed to make a timely switch from film to digital photography (The Economist, 2012a). Similar examples include Xerox, which could not adapt to a world dominated by digital imaging, or NCR (National Cash Register), which was once a dominant player in computer hardware and software but failed to adjust itself to personal computers and ended up relegated to ATM machines (The Economist, 2012b). Common features of these examples are the underestimation of the magnitude of technological change, as well as the reluctance to abandon a well-established technology in order to enter a potentially more profitable market regime. Indeed, decisionmakers often exhibit risk aversion, which hobbles any effort for technological change, while market-regime asymmetries combined with economic and technological uncertainty complicate technology-switching decisions. Although the impact of technological uneertainty on the propensity to invest in technological innovations has been analysed extensively under risk neutrality (Huisman \& Kort, 2004; Chronopoulos \& Siddiqui, 2015), how attitudes towards risk influence investment and operational decisions under price and technological uncertainty has not been thoroughly studied yet.

Indeed, although empirical research has studied the implications of market incompleteness for the development and adoption of innovations in nascent markets (Ang, 2014), how market incompleteness influences attitudes towards risk, and, in turn, incentives for technology adoption remains an open question. Therefore, we develop a real options framework in order to explore how economic and technological uncertainty impact incentives for technological change, taking into account a decisionmaker's risk preferences as well as her discretion over the technology-adoption strategy. The latter is implemented by assuming that the decisionmaker may invest in either each technology that becomes available (compulsive) or delay investment until a new technology arrives and then either invest in the older (laggard) or the newer technology (leapfrog). Thus, the novelty of this work is that, by combining attitudes towards risk with various market uncertainties, it is possible to analyse how their interaction impacts not only the dominant technology-adoption strategy, but also, within each strategy, the optimal investment and operational decisions. In fact, this work takes into account a wide range of attitudes towards risk by considering both risk-averse and riskseeking behaviour. Although the former is more plausible, evidence of the latter can be found in at 
least two situations that are particularly relevant to technology adoption. For example, it may be common to invest in projects with high upside potential, e.g., startups, rather than in conservative ones, with the expectation of making a high return in just a small subset of the selected projects (Nawrocki, 2002). Also, firms that are underperforming their peers might distantiate themselves from the competition by adopting a new technology, thus acknowledging that only a bold move may salvage an otherwise doomed company (Bowman, 1982 and Bromiley, 1991).

Additionally, despite the extensive literature on sequential investment in improved versions of a single technology (Parente, 1994), the implications of technological uncertainty for investment in technological breakthroughs have not been analysed thoroughly yet (Doraszelski, 2004). Therefore, we assume that once an innovation takes place at a random point/in time, it not only creates a new market regime but also reduces the profitability of the existing one. Within this context, a decisionmaker has the flexibility to abandon the existing regime and invest in the new one. Consequently, the contribution of our work is threefold. First, we develop an regime-switching, utility-based framework for sequential investment under uncertainty and operational flexibility in order to derive optimal investment and operational thresholds. Second, we show how attitudes towards risk interact with price and technological uncertainty to affect not only the optimal regimeswitching strategy, but also, within each strategy, the optimal investment and operational decisions. Third, we provide managerial insights for investment and operational decisions based on analytical and numerical results.

We proceed by discussing some related work in Section 2 and introduce assumptions and notation in Section 3. The problem of investment in a new regime is addressed in Section 4.1, while, in Section 4.2, we tackle the problem of abandoning an old regime in order to invest in a new one, and, in Section 4.3, we analyse the problem of investment under regime switching. In Section 5 , we analyse the choice between two alternative market regimes, and, in Section 6 , we present a comparison of the different technology-adoption strategies. Section 7 provides numerical examples for each case and examines the effects of uncertainty and risk aversion on the optimal investment and operational thresholds. Section 8 concludes and offers directions for future research.

\section{Literature Review}

The seminal work of McDonald \& Siegel $(1985,1986)$ and Dixit \& Pindyck (1994) has spawned a substantial literature in the area of investment under uncertainty. However, most of this literature is developed on the premise that decisionmakers are risk neutral and hold a perpetual option to invest, facing a single form of uncertainty. Consequently, analytical models that explore the implications 
of risk aversion as well as the combined impact of different types of uncertainties on investment and operational decisions remain somewhat underdeveloped. The two main methods for addressing the canonical real options problem are contingent claims and dynamic programming. The former assumes that either markets are complete or that the project's unique risk can be perfectly hedged. Consequently, it cannot be applied to projects with idiosyncratic risk that cannot be diversified, as is the case with most technology adoption problems, or, more generally, when markets do not have substantially developed financial instruments. In these cases, the dynamic programming approach can still be applied as it uses a subjective discount rate, and, therefore, it can be used to maximise the expected discounted utility of the lifetime profits of a risk-averse decisionmaker.

Examples of early work in the area of investment under technological uncertainty include Balcer \& Lippman (1984), who model technological uncertainty via a discrete semi-Markov process and find that a higher rate of innovation tends to delay technology adoption. Grenadier \& Weiss (1997) consider a firm that, in the light of technological uncertainty, may either adopt each technology that becomes available (compulsive) or postpone investment until an innovation takes place and then either adopt an older (laggard) or a newer technology (leapfrog). They find that, depending on technological uncertainty, a firm may adopt an available technology even if more valuable innovations may occur in the future, while future decisjons on technology adoption are path dependent. Farzin et al. (1998) develop an analytical framework for sequential investment in technological innovations that follow a Poisson process, using dynamic programming. They find that the investment rule derived via the real options theory coincides with the net present value (NPV) criterion for all but the last investment. By contrast, Doraszelski (2001) identifies an error in Farzin et al. (1998) and shows that, compared to the NPV criterion, a firm will defer the adoption of a technology when it takes the value of waiting into account.

In the same line of work, Bethuyne (2002) considers a firm that holds a number of technology investment options and identifies an ambiguous effect, whereby technological improvement induces replacement but the prospect of further improvements slows down the replacement process. In addition, a decrease in the number of remaining technology switches raises the value of each investment option. Huisman \& Kort (2003) replace technological uncertainty in the framework of Grenadier \& Weiss (1997) with game-theoretic considerations, while Huisman \& Kort (2004) develop an analytical framework for duopolistic competition allowing for technological uncertainty. Their results indicate that, when technology upgrading is not optimal, a second-mover advantage arises when producing with the new technology in the future leads to a higher payoff than the current temporary monopoly profits. Doraszelski (2004) introduces a distinction between technological breakthroughs 
and engineering refinements. He shows how firms do not necessarily wait for a future technological breakthrough, but instead may delay the adoption of a new technology until it has been sufficiently refined. More recently, Koussis et al. (2013) model market uncertainty via a jump-diffusion process that allows for multiple classes of jumps, and, in turn, for the flexibility to model different independent risks affecting a firm, e.g., entry of differentiated products and technological uncertainty. Although technological uncertainty is a crucial feature of emerging technologies, the scope of the aforementioned papers is limited as they assume risk neutrality, thereby ignoring the implícations of risk aversion due to technical risk for investment and operational decisions.

Examples of analytical frameworks that incorporate risk aversion into the dynamics of investment decisions include Henderson \& Hobson (2002), who extend the real options approach to pricing and hedging assets by taking the perspective of a risk-averse decisionmaker facing incomplete markets. They introduce a second risky asset on which no trading is allowed in the framework of Merton (1969) and address the problem of pricing and hedging this random payoff. Henderson (2007) addresses the problem of irreversible investment under uncertainty taking the perspective of a risk-averse decisionmaker. Although part of the uncertainty associated with the investment payoff can be hedged via a risky asset that is correlated with the investment payoff and a risk-free bond, the remaining risk is idiosyncratic. Results indicate that higher risk aversion or lower correlation between the project value and the hedging asset lowers both the option value and the investment threshold. In particular, there is a parameter region where the option is exercised (never exercised) under the assumption of complete (incomplete) markets. Huggonier \& Morellec (2013) use an optimal stopping-time approach to allow for the decisionmaker's risk aversion to be incorporated via a constant relative risk aversion (CRRA) utility function. Their framework is based on a closedform expression for the expected discounted utility of stochastic cash flows derived by Karatzas \& Shreve (1999). Results indicate that risk aversion erodes the value of a project and lowers the likelihood of investment. In the same line of work, Chronopoulos et al. (2011, 2013, and 2014) analyse the impact of risk aversion on investment allowing for operational flexibility, discretion over capacity, and competition. While risk aversion is an important attribute of investment decisions within emerging markets and the R\&D-based sector of the economy, the aforementioned papers ignore the sequential nature of these investments, and, particularly, how technological uncertainty may impact investment and operational decisions of risk-averse decisionmakers.

The problem of technology switching can be assimilated to a choice between mutually exclusive projects. Dixit (1993) analyses this problem under price uncertainty and finds that increasing returns and uncertainty make it optimal to wait in order to invest in the project with the highest 
expected NPV. Décamps et al. (2006) extend Dixit (1993) by providing parameter restrictions under which the optimal investment strategy is not a trigger strategy and the optimal investment region is dichotomous. The analytical framework of Décamps et al. (2006) was subsequently adopted by Fleten et al. (2007) and Siddiqui \& Fleten (2010). The former, model investment in wind turbines taking the perspective of an investor who must choose among discrete alternatives and has discretion over both the time of investment and the size of the project. The latter, analyse how a firm may proceed with staged commercialization and deployment of competing alternative energy technologies. Hagspiel et al. (2013) consider a risk-neutral, price-setting firm that faces both technological change and a declining profit stream. The firm can either abandon the current project, suspend operations temporarily, or invest in a new technology. Their results indicate that with (without) discretion over capacity, the relationship between the optimal investment threshold and uncertainty is monotonic (non-monotonic). In addition, the firm suspends operations only when uncertainty is high and the market for the innovative product is very attractive. However, apart from risk neutrality, a common feature of these models is that they ignore technological uncertainty as the availability of the alternative projects is not subject to a probability distribution.

More pertinent to our analysis is the framework of Alvarez \& Stenbacka (2004), who implement attitudes towards risk via a hyperbolic absolute risk aversion (HARA) utility function and develop an analytical framework for regime-switching under price uncertainty. More specifically, they consider the problem of optimal switching from one stochastic cash flow representation to another, which, contrary to Alvarez \& Stenbacka (2001), implies a structural change in the project's cash flows in terms of a change in volatility while keeping the drift constant. They conclude that increasing volatility does not necessarily postpone investment. In fact, for a risk-seeking decisionmaker, they find that the opposite is true. However, although they analyse the implications of attitudes towards risk for regime-switching decisions, they assume no uncertainty in the arrival of innovations, thereby ignoring the implications of technological uncertainty for investment and operational decisions. In turn, this limits any additional insights in relation to the optimal technology-adoption strategy (Grenadier \& Weiss, 1997; Décamps et al., 2006; Chronopoulos \& Siddiqui, 2015).

In this paper, we assume that a decisionmaker has the option to invest sequentially in emerging technologies under price and technological uncertainty. We capture these features by developing a utility-based, regime-switching framework, where the price process follows a Markov-modulated geometric Brownian motion (GBM) and innovations follow a Poisson process. Once an innovation occurs, a new, more attractive, market regime emerges, while the attractiveness of the incumbent regime is reduced. In order to enter the new market regime, the decisionmaker must abandon the 
existing one. Consequently, this approach enables a comparison with models that either do not allow for operational flexibility (Alvarez \& Stenbacka, 2004) or may allow for suspension and resumption options, yet assume symmetric regimes (Chronopoulos et al., 2011). We find that if market regimes are asymmetric, then greater price uncertainty and risk aversion may induce earlier abandonment of a mature technology. The former result is in contrast to the traditional real options intuition, which states that greater price uncertainty tends to delay investment and operational decisions by increasing the value of waiting. Additionally, the latter result is contrary to Chronopoulos et al. (2011), who find that greater risk aversion delays the temporary suspension of a project prior to permanent resumption. Furthermore, we determine the optimal technology-adoption strategy and find that, while risk aversion typically favours a compulsive strategy, a leapfrog strategy may dominate even when the decisionmaker is risk averse, provided that both the output price and the rate of innovation are high.

\section{Assumptions and Notation}

We consider a decisionmaker with a sequence of perpetual options to invest in projects of infinite lifetime facing price and technological uncertainty. Time is continuous and denoted by $t \geq 0$, while $(\Omega, \mathcal{F}, \mathbb{P})$ is a complete probability space and $\left\{\mathcal{F}_{t} \subset \mathcal{F}, t \in[0, \infty)\right\}$ is a $\sigma$-algebra contained by $\mathcal{F}$, increasing in $t$, and right continuous. Intuitively, $\mathcal{F}_{t}$ represents the information that is available at time $t$. The projects' exogenous output price, $P_{t}^{(k)}$, where $k \in\{1,2,3\}$, follows a Markov-modulated geometric Brownian motion (GBM) that is described in (1), where $\mu_{k}$ is the annual growth rate, $\sigma_{k}$ is the annual volatility, and $d Z_{t}$ is the increment of the standard Brownian motion.

$$
d P_{t}^{(k)}=\mu_{k} P_{t}^{(k)} d t+\sigma_{k} P_{t}^{(k)} d Z_{t}, \quad P_{0}^{(k)} \equiv P>0
$$

The arrival of innovations follows a Poisson process $\left\{M_{t}, t \geq 0\right\}$, which is independent of $P_{t}^{(k)}$. Thus, the probability of an innovation occurring within an infinitesimal time interval $d t$, is $\nu d t$, where $\nu$ is the intensity of the Poisson process. Once an innovation takes place, the market parameters for the existing téchnology switch from regime $k=1$ to regime $k=2$, while a new market regime, $k=3$, emerges. The implications of technological uncertainty for the growth rate of each technology within the corresponding market regime are reflected in the inequality $\mu_{3}>\mu_{1}>\mu_{2}$. This implies that: (i) the emergence of a new market regime reduces the attractiveness of an old one $\left(\mu_{1}>\mu_{2}\right)$ and (ii) that the new market regime reflects the arrival of a superior technology, and, therefore, presents a growth rate that is greater than that of the first regime $\left(\mu_{3}>\mu_{1}\right)$, in which the first technology was initially dominating the market. Additionally, although technological breakthroughs tend to 
reduce the attractiveness of mature technologies, the volatility associated with a new market regime may be initially greater than the one in the existing regime. Therefore, we consider both $\sigma_{3}>\sigma_{2}$ and $\sigma_{3}<\sigma_{2}$.

The decisionmaker's preferences are described by the functional indicated in (2), where $U(\cdot)$ denotes the utility function and $\rho \geq \mu$ the subjective discount rate. We also denote the risk-free rate by $r$ and assume that the utility function is increasing and may be either convex or concave.

$$
P \longmapsto \int_{0}^{\infty} e^{-\rho t} U\left(P_{t}^{(k)}\right) d t
$$

Additionally, we assume that $U(\cdot)$ satisfies the integrability condition, i.e., $\left.E \int_{0}^{\infty} e^{-\rho t} U^{(}\left(P_{t}^{(k)}\right) d t \mid \mathcal{F}_{0}\right]$ $<\infty$, and, in line with Henderson (2007) and Hugonnier and Morellee (2013), we assume that the subjective discount rate, $\rho$, is defined exogenously, and, therefore, is not affected by the decisionmaker's risk preferences, which are reflected solely via the utility function, $U(\cdot)$. Consequently, our analysis can accommodate a wide range of utility functions, e.g., hyperbolic absolute risk aversion (HARA), constant absolute risk aversion (CARA), and constant relative risk aversion (CRRA) utility functions. Here, we apply an HARA utility function, which is indicated in (3). Although standard economic theory assumes that decisionmakers are typically risk averse (Pratt, 1964), for the purposes of the analysis we assume that $\gamma \in[0.5,1.5]$. Thus, we explore the implications of both risk-averse $(0.5<\gamma<1)$ and risk-seeking $(1<\gamma<1.5)$ behaviour, thereby allowing for comparisons with both Chronopoulos et al. (2011) and Hugonnier and Morellec (2013).

$$
U(P)=\frac{P^{\gamma}}{\gamma}, \quad \gamma>0
$$

We let $i=0,1$ denote the state of a technology in terms of being active $(i=1)$ or inactive $(i=0)$. Also, we let $\tau_{i}^{(k)}$ denote the time of investment $(i=1)$ or abandonment $(i=0)$ of a technology in regime $k$ and $p_{i}^{(k)}$ denote the corresponding optimal price threshold. The variable operating cost of an old technology is denoted by $c$, while a new technology is more efficient, and, therefore, we assume that it entails no operating cost. Additionally, the fixed and irrecoverable cost of investment in or abandonment of a technology in regime $k$ is denoted by $I_{1}^{(k)}$ and $I_{0}^{(k)}$, respectively, while the output under regime $k$ is $D_{k}$. The expected utility of an active project is denoted by $\Phi_{i}^{(k)}(\cdot)$ and the maximised expected NPV from investment or abandonment by $F_{i}^{(k)}(\cdot)$. For example, the time of investment in or abandonment of the first technology in the second regime is denoted by $\tau_{1}^{(2)}$ and $\tau_{0}^{(2)}$, respectively, while the corresponding optimal price thresholds are denoted by $p_{1}^{(2)}$ and $p_{0}^{(2)}$, respectively.

As we will see in Section 5, in order to have a tradeoff between an old and a new technology (market regime), we assume that at the point, $\widetilde{p}$, where the expected utilities of the profits from 
each technology are equal, we have $\Phi_{1}^{(2)}(\widetilde{p})=\Phi_{1}^{(3)}(\widetilde{p})>0$. Otherwise, only the new technology (market regime) presents a viable investment opportunity, as its expected utility is always greater than that of the old technology for all the positive values of its range (Décamps et al., 2006). Intuitively, a new technology may produce a greater output compared to an old one, yet is much more capital intensive. Note that, under risk neutrality, this condition simplifies to $\frac{D_{3}}{I_{1}^{(3)}}<\frac{D_{2}}{I_{1}^{(2)}}$, as in Chronopoulos and Siddiqui (2015).

\section{Compulsive Strategy}

\subsection{Regime 3}

The value function within each market regime is determined via backward induction. Therefore, we begin by assuming that, after having just exited the second regime, the decisionmaker is initially in an inactive state and considers investing directly in the third one. Since there is no operating cost associated with the new technology, it remains active forever after investment. Following the approach of Huggonier \& Morellec (2013), we decompose the cash flows of the project into disjoint time intervals. Hence, we assume that the capital required for the realisation of the project is initially invested in a certificate of deposit and earns a risk-free rate up to time $\tau_{1}^{(3)}$. At time $\tau_{1}^{(3)}$, the decisionmaker swaps this risk-free cash flow for the risky cash flows that the project generates, as in Figure 1.

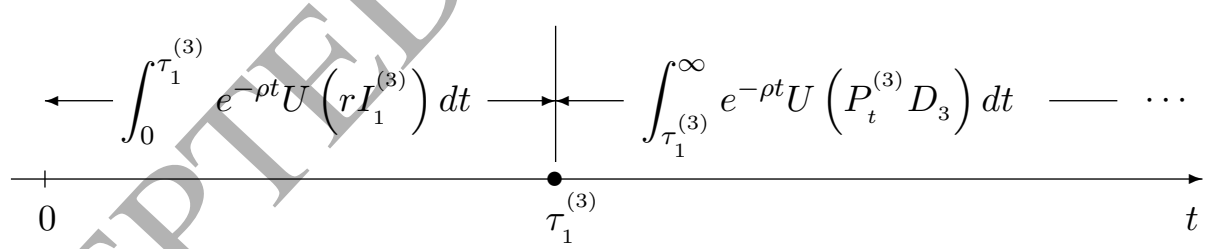

Figure 1: Investment in regime 3

The objective of the decisionmaker is to determine the investment policy that maximises the timezero expected discounted utility of all the cash flows of the project. Thus, the decisionmaker must select the time $\tau_{1}^{(3)}$ that solves problem $(4)$, where $\mathbb{E}\left[\cdot \mid \mathcal{F}_{t}\right]$ is the expectation operator that is conditional on the information at time $t$ and $\mathcal{S}$ is the set of stopping times of the filtration generated by $P_{t}^{(3)}$.

$$
F_{1}^{(3)}(P)=\sup _{\tau_{1}^{(3)} \in \mathcal{S}} \mathbb{E}\left[\int_{0}^{\tau_{1}^{(3)}} e^{-\rho t} U\left(r I_{1}^{(3)}\right) d t+\int_{\tau_{1}^{(3)}}^{\infty} e^{-\rho t} U\left(P_{t}^{(3)} D_{3}\right) d t \mid \mathcal{F}_{0}\right]
$$

Next, we decompose the first integral on the right-hand side of (4) and apply the law of iterated expectations and the strong Markov property of the GBM. The latter, states that price values after 
time $\tau_{1}^{(3)}$ are independent of the values before $\tau_{1}^{(3)}$ and depend only on the value of the process at $\tau_{1}^{(3)}$. Thus, the optimisation objective (4) can be written as in (5). The first term on the right-hand side of (5) is the stochastic discount factor, while, the second term, is the expected utility of the active project at $\tau_{1}^{(3)}$.

$$
F_{1}^{(3)}(P)=\sup _{\tau_{1}^{(3)} \in \mathcal{S}} \mathbb{E}\left[e^{-\rho \tau_{1}^{(3)}} \mid \mathcal{F}_{0}\right] \times \mathbb{E}\left[\int_{0}^{\infty} e^{-\rho t}\left[U\left(P_{t}^{(3)} D_{3}\right)-U\left(r I_{1}^{(3)}\right)\right] d t \mid \mathcal{F}_{\tau_{1}^{(3)}}\right]
$$

From Theorem 9.18 of Karatzas and Shreve (1999), we can determine the analytical expression for the expected utility of cash flows that follow GBM under a HARA utility function. Hence, the expected utility of the profits from immediate investment in the third regime is indicated in (6), where $\mathcal{A}^{(k)}=\frac{\beta_{1 k} \beta_{2 k}}{\rho\left(\gamma-\beta_{1 k}\right)\left(\gamma-\beta_{2 k}\right)}$ and $\beta_{\ell k}, \ell=1,2$, are the roots of the quadratic $\frac{1}{2} \sigma_{k}^{2} \beta(\beta-1)+\mu_{k} \beta-\rho=$ $0, k=1,2,3$.

$$
\Phi_{1}^{(3)}(P)=\mathbb{E}\left[\int_{0}^{\infty} e^{-\rho t}\left[U\left(P_{t}^{(3)} D_{3}\right) d t-U\left(r I_{1}^{(3)}\right)\right] \mid \mathcal{F}_{0}\right]=\mathcal{A}^{(3)} U\left(P D_{3}\right)-\frac{U\left(r I_{1}^{(3)}\right)}{\rho}
$$

The decisionmaker's optimisation objective can be expressed equivalently as in (7). The top part on the right-hand side of (7) represents the value of the option to invest in the third regime and the bottom part is the expected utility of the active project.

$$
F_{1}^{(3)}(P)= \begin{cases}A_{1}^{(3)} P^{\beta_{13}} & , P<p_{1}^{(3)} \\ \Phi_{1}^{(3)}(P) & , P \geq p_{1}^{(3)}\end{cases}
$$

By applying value-matching and smooth-pasting conditions between the two branches of (7), we can determine the analytical expression for the endogenous constant, $A_{1}^{(3)}$, and the required investment threshold, $p_{1}^{(3)}$, as indicated in (8). Notice that, although the investment threshold is usually expressed in terms of $\beta_{1 k}$, it is more expedient to use $\beta_{2 k}$ here, taking into account that $\beta_{1 k} \beta_{2 k}=$ $-\frac{2 \rho}{\sigma_{k}^{2}}$

$$
A_{1}^{(3)}=\left(\frac{1}{p_{1}^{(3)}}\right)^{\beta_{13}} \Phi_{1}^{(3)}\left(p_{1}^{(3)}\right) \text { and } p_{1}^{(3)}=\frac{r I_{1}^{(3)}}{D_{3}}\left(\frac{\beta_{23}-\gamma}{\beta_{23}}\right)^{\frac{1}{\gamma}}
$$

The second-order sufficiency condition (SOSC) requires the objective function to be concave at $p_{1}^{(3)}$, which we show in Proposition 1. All proofs can be found in the Appendix.

Proposition 1. The objective function $F_{1}^{(3)}(P)$ is strictly concave at $p_{1}^{(3)}$.

As shown in Proposition 2, greater price uncertainty raises the required investment threshold by increasing the opportunity cost of investment, and, in turn, the value of waiting. Additionally, if 
$U(\cdot)$ is concave $(\gamma<1)$, then a decrease in $\gamma$ raises risk aversion and increases the incentive to postpone investment by reducing the expected utility of the active project. By contrast, if $U(\cdot)$ is convex $(\gamma>1)$, then an increase in $\gamma$ lowers risk aversion and accelerates investment by increasing the expected utility of the active project.

Proposition 2. $\forall \sigma_{3}>0$ and $\forall \gamma \in[0.5,1.5]$ we have $\frac{\partial p_{1}^{(3)}}{\partial \sigma_{3}}>0$ and $\frac{\partial p_{1}^{(3)}}{\partial \gamma}<0$.

\subsection{Regime 2}

Here, we step back and assume that the decisionmaker holds an option to invest in the second regime with an embedded option to abandon it permanently, should the output price drop, and subsequently invest in the third regime. Notice that, by abandoning the second regime, the decisionmaker foregoes the revenues of the active project, yet recovers the salvageable operating cost. Hence, the expected utility from immediate abandonment of the second regime is indicated in (9).

$$
\begin{aligned}
\Phi_{0}^{(2)}(P) & =\mathbb{E}\left[\int_{0}^{\infty} e^{-\rho t}\left[U\left(c D_{2}-r I_{0}^{(2)}\right)-U\left(P_{t}^{(2)} D_{2}\right) d t\right] \mid \mathcal{F}_{0}\right] \\
& =\frac{U\left(c D_{2}-r I_{0}^{(2)}\right)}{\rho}-\mathcal{A}^{(2)} U\left(P D_{2}\right)
\end{aligned}
$$

Next, we assume that the project is operating in the second regime and determine the expected value of the option to abandon it, which is described in (10). The top part on the right-hand side of (10) is the expected value of the option to abandon the second regime, while the bottom part consists of the expected utility of the active project in an abandoned state (first term) and the expected value of the option to invest in the third regime (second term). The latter is indicated in the top part of $(7)$.

$$
F_{0}^{(2)}(P)= \begin{cases}A_{0}^{(2)} P^{\beta_{22}} & , P<p_{0}^{(2)} \\ \Phi_{0}^{(2)}(P)+F_{1}^{(3)}(P) & , P \geq p_{0}^{(2)}\end{cases}
$$

By applying yalue-matching and smooth-pasting conditions between the two branches of (10), we can determine the endogenous constant, $A_{0}^{(2)}$, and the required abandonment threshold, $p_{0}^{(2)}$, numerically. However, if the embedded option to invest in the third regime is not available, then these expressions can be obtained analytically. Indeed, in the absence of the option to invest in the third regime, the value of the option to abandon the second one is denoted by $\bar{F}_{0}^{(2)}(P)$ and is indicated in (11).

$$
\bar{F}_{0}^{(2)}(P)= \begin{cases}\bar{A}_{0}^{(2)} P^{\beta_{22}} & , P<\bar{p}_{0}^{(2)} \\ \Phi_{0}^{(2)}(P) & , P \geq \bar{p}_{0}^{(2)}\end{cases}
$$


The analytical expressions of the endogenous constant, $\bar{A}_{0}^{(2)}$, and the required abandonment threshold, $\bar{p}_{0}^{(2)}$, are indicated in (12).

$$
\bar{A}_{0}^{(2)}=\left(\frac{1}{\bar{p}_{0}^{(2)}}\right)^{\beta_{12}} \Phi_{0}^{(2)}\left(\bar{p}_{0}^{(2)}\right) \quad \text { and } \quad \bar{p}_{0}^{(2)}=\frac{c D_{2}-r I_{0}^{(2)}}{D_{2}}\left(\frac{\beta_{12}-\gamma}{\beta_{12}}\right)^{\frac{1}{\gamma}}, c D_{2}>r I_{0}^{(2)}
$$

To ensure the existence of a local maximum, the SOSC has to be verified. This is shown in Proposition 3 for the case in which the embedded option to invest in the third regime is not available.

Proposition 3. The objective function $\bar{F}_{0}^{(2)}(P)$ is strictly concave at $\bar{p}_{0}^{(2)}$.

As shown in Proposition 4, greater price uncertainty in the second regime increases the incentive to postpone abandonment. Intuitively, this happens because the decisionmaker would not want to abandon the project permanently due to a temporary downturn, which is more likely when uncertainty is high. Also, if $U(\cdot)$ is concave (convex), then lower (greater) $\gamma$ raises (lowers) the required abandonment threshold. Indeed, if $\gamma<1$, then lower $\gamma$ decreases the expected utility of the project, thereby increasing the incentive to abandonit. By contrast, if $\gamma>1$, then greater $\gamma$ implies a more risk-seeking behaviour, which increases the incentive to postpone abandonment.

Proposition 4. $\forall \sigma_{2}>0$ and $\forall \gamma \in[0.5,1.5]$ we have $\frac{\partial \bar{p}_{0}^{(2)}}{\partial \sigma_{2}}<0$ and $\frac{\partial \bar{p}_{0}^{(2)}}{\partial \gamma}<0$.

In order to analyse the impact of $\gamma$ on the required abandonment threshold when the option to invest in the third regime is taken into account, we express the maximised value of the option to abandon the second regime as in (13).

$$
F_{0}^{(2)}(P)=\left(\frac{P}{p_{0}^{(2)}}\right)^{\beta_{22}}\left[\Phi_{0}^{(2)}\left(p_{0}^{(2)}\right)+F_{1}^{(3)}\left(p_{0}^{(2)}\right)\right]
$$

By applying the first-order necessary condition (FONC) to (13), we can express the optimal abandonment rule in (14) by equating the marginal benefit (MB) of accelerating abandonment (left-hand side) to the marginal cost (MC) (right-hand side). Notice that both terms on the left-hand side of (14) are positive, thereby indicating that abandoning operations at a higher price level, i.e., more quickly, increases the expected utility of both the revenues from investing in the third regime (first term) and the salvageable operating cost (second term). Also, the first term on the right-hand side of (14) is positive and corresponds to the MC of killing the revenues of the project at a higher price level, while, the second term, is also positive and corresponds to the increase in the MC from speeding up abandonment. This term represents the increase in the opportunity cost from waiting 
less, thereby forgoing information. The last term represents the increase in the expected utility of the cost from investing in the third regime.

$$
\begin{aligned}
& \left(\beta_{13}-\beta_{22}\right)\left(\frac{p_{0}^{(2)}}{p_{1}^{(3)}}\right)^{\beta_{13}} \mathcal{A}^{(3)} U\left(p_{1}^{(3)} D_{3}\right)-\frac{\beta_{22}}{\rho} U\left(c D_{2}-r I_{0}^{(2)}\right) \\
& =\gamma \mathcal{A}^{(2)} U\left(p_{0}^{(2)} D_{2}\right)-\beta_{22} \mathcal{A}^{(2)} U\left(p_{0}^{(2)} D_{2}\right)+\left(\beta_{13}-\beta_{22}\right)\left(\frac{p_{0}^{(2)}}{p_{1}^{(3)}}\right)^{\beta_{13}} \frac{U\left(r I_{1}^{(3)}\right)}{\rho}
\end{aligned}
$$

Notice that the embedded option to switch to the third regime raises the incentive to abandon the second one, and, as a result, $p_{0}^{(2)}>\bar{p}_{0}^{(2)}$. This happens because at $p_{1}^{(3)}$, the expected utility of the revenues from investing in the third regime is greater than the expected utility of the investment cost, and, as a result, the MB of accelerating abandonment increases by more than the MC, thereby raising the incentive to abandon the second regime. As Proposition 5 indicates, this effect is further pronounced as $D_{3}$ increases. This implies that a greater market share in the third regime lowers the attractiveness of the second one, thereby increasing the incentive to abandon it.

Proposition 5. A ceteris paribus increase in $D_{3}$ raises $p_{0}^{(2)}$.

Proposition 6 indicates that greater price uncertainty in the second regime lowers the required abandonment threshold, $p_{0}^{(2)}$. This extends the result of Proposition 4 by taking into account the embedded option to invest in the third regime. In line with Proposition 4, the decisionmaker is more reluctant to abandon the second regime due to a temporary downturn, which is more likely when uncertainty is high. Interestingly, however, greater uncertainty in the third regime accelerates the abandonment of the second one. This is contrary to the conventional real options intuition, which indicates that greater uncertainty raises the incentive to postpone abandonment. This seemingly counter-intuitive result happens because greater price uncertainty in the third regime raises the expected value of the corresponding investment option, thereby increasing the decisionmaker's incentive to abandon the second regime in order to have the option to invest in the third one.

Proposition 6. A ceteris paribus increase in $\sigma_{3}\left(\sigma_{2}\right)$ increases (decreases) $p_{0}^{(2)}$.

Having derived the expected value of the option to abandon the second regime, we step back and determine the value of the option to invest in the second regime with an embedded abandonment option. This is indicated in (15), where the top part on the right-hand side of (15) is the expected value of the investment opportunity and the bottom part is the expected utility of the active project. The latter consists of the expected utility of the profits from operating the first technology in the second regime (first two terms) and the option to abandon it inclusive of the embedded option to 
invest in the third regime (third term). By applying value-matching and smooth-pasting conditions between the two branches of (15), we can determine $A_{1}^{(2)}$ and $p_{1}^{(2)}$, numerically.

$$
F_{1}^{(2)}(P)= \begin{cases}A_{1}^{(2)} P^{\beta_{21}} & , P<p_{1}^{(2)} \\ \mathcal{A}^{(2)} U\left(P D_{2}\right)-\frac{U\left(r I_{1}^{(2)}+c D_{2}\right)}{\rho}+F_{0}^{(2)}(P) & , P \geq p_{1}^{(2)}\end{cases}
$$

\subsection{Regime 1}

The expected utility of the active project in the first regime is indicated in (16). The first term on the right-hand side of (16) is the instantaneous utility of the profits in the first regime. As the second term indicates, within an infinitesimal time interval $d t$, an innovation may take place with probability $\nu d t$ and the operation of the first technology will continue in the second regime. In this case, the decisionmaker also receives the option to abandon the second regime and invest in the third one. By contrast, as the third term indicates, with probability $1-\nu d t$ no innovation will take place and the first technology will continue to operate in the first regime.

$$
\begin{aligned}
\Phi_{1}^{(1)}(P)=\left[U\left(P D_{1}\right)-U\left(c D_{1}+r I_{1}^{(1)}\right)\right] d t & +(1-\rho d t) \nu d t \mathbb{E}\left[\Phi_{1}^{(2)}(P+d P)+F_{0}^{(2)}(P+d P) \mid \mathcal{F}_{0}\right] \\
& +(1-\rho d t)(1-\nu d t) \mathbb{E}\left[\Phi_{1}^{(1)}(P+d P) \mid \mathcal{F}_{0}\right]
\end{aligned}
$$

By expanding the right-hand side of (16) using Itô's lemma and solving the resulting ordinary differential equation, we obtain the expression for $\Phi_{1}^{(1)}(P)$, which is indicated in (17). Note that $\delta_{1}, \delta_{2}$ are the roots of the quadratic $\frac{1}{2} \sigma_{2}^{2} \delta(\delta-1)+\mu_{1} \delta-(\rho+\nu)=0, \mathcal{D}=\frac{\delta_{1} \delta_{2}}{(\rho+\nu)\left(\gamma-\delta_{1}\right)\left(\gamma-\delta_{2}\right)}$, and $B_{0}^{(1)}=-\nu A_{0}^{(2)} /\left(\frac{1}{2} \sigma_{1}^{2} \beta_{22}\left(\beta_{22}-1\right)+\mu_{1} \beta_{22}-(\rho+\nu)\right)$.

$$
\Phi_{1}^{(1)}(P)=\mathcal{D} U\left(P D_{1}\right)+\nu \mathcal{D} \mathcal{A}^{(2)} U\left(P D_{2}\right)-\frac{U\left(c D_{1}+r I_{1}^{(1)}\right)}{\rho+\nu}-\frac{\nu U\left(c D_{2}+r I_{1}^{(2)}\right)}{\rho(\rho+\nu)}+B_{0}^{(1)} P^{\beta_{22}}
$$

Also, notice that if $\nu=0$, then the second, fourth, and fifth term in (17) are zero. Intuitively, $\nu=0$ implies that no regime switching will take place, and, as a result, the first technology will continue to operate for ever in the first regime. By contrast, $\lim _{\nu \rightarrow \infty} \Phi_{1}^{(1)}(P)=\Phi_{1}^{(2)}(P)$ since $\lim _{\nu \rightarrow \infty} \nu \mathcal{D}=1$ and $\lim _{\nu \rightarrow \infty} \mathcal{D}=0$.

Next, the dynamics of the option to invest in the first regime are described in (18). The first term on the right-hand side of (18) indicates that, while waiting to invest in the first regime, an innovation may take place with probability $\nu d t$ and the decisionmaker will receive the value function $F_{1}^{(2)}(P)$. By contrast, with probability $1-\nu d t$ no innovation will take place and the decisionmaker will continue to hold the value function $F_{1}^{(1)}(P)$.

$$
F_{1}^{(1)}(P)=(1-\rho d t)\left[\nu d t \mathbb{E}\left[F_{1}^{(2)}(P+d P) \mid \mathcal{F}_{0}\right]+(1-\nu d t) \mathbb{E}\left[F_{1}^{(1)}(P+d P) \mid \mathcal{F}_{0}\right]\right]
$$


By expanding the right-hand side of (18) using Itô's lemma we obtain (19), which must be solved together with (20), i.e., the differential equation for the value of the option to invest in the second regime.

$$
\begin{aligned}
\frac{1}{2} \sigma_{1}^{2} P^{2} F_{1}^{(1)^{\prime \prime}}(P)+\mu_{1} P F_{1}^{(1)^{\prime}}(P)-(\rho+\nu) F_{1}^{(1)}(P)+\nu F_{1}^{(2)}(P) & =0 \\
\frac{1}{2} \sigma_{2}^{2} P^{2} F_{1}^{(2)^{\prime \prime}}(P)+\mu_{2} P F_{1}^{(2)^{\prime}}(P)-\rho F_{1}^{(2)}(P) & =0
\end{aligned}
$$

Hence, the value of the option to invest in the first regime is obtained by solving the set of differential equations (19)-(20) and is described in (21), where $A_{1}^{(1)}$ and $p_{1}^{(1)}$ are obtained numerically via value-matching and smooth-pasting conditions between the two branches of $(21)$, while $C_{1}^{(1)}=$ $-\nu A_{1}^{(2)} /\left(\frac{1}{2} \sigma_{1}^{2} \beta_{21}\left(\beta_{21}-1\right)+\mu_{1} \beta_{21}-(\rho+\nu)\right)$.

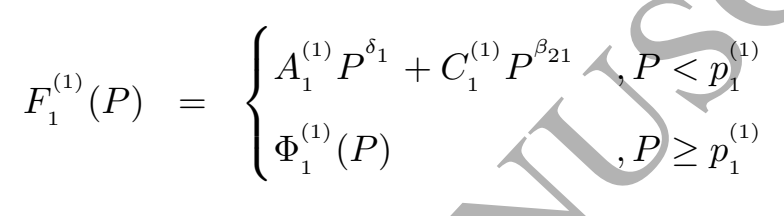

\section{Leapfrog versus Laggard Strategy}

It is possible that a more attractive market regime emerges while waiting to invest in the existing one, thus replacing the initial investment option with the option to choose between two alternative regimes. Here, we assume that the decisionmaker would not want to invest in an existing regime before comparing it to the next one. Notice that in order to have a tradeoff between an older an a newer market regime, the expected utility of the active project at the point of indifference, $\widetilde{p}$, between the two regimes must be positive. Intuitively, this implies that the demand under the new market regime is greater than that under the old one, yet, the investment cost is much greater.

We begin by assuming that both market regimes are available. Thus, the decisionmaker can choose to invest directly in either the third (leapfrog) or $(\vee)$ the second regime (laggard) with an embedded option to abandon it and then switch to the third one. By extending the framework of Décamps et al. (2006) to allow for attitudes towards risk, we obtain the value function under a leapfrog/lággard strategy, which is described in (22).

$$
F_{1}^{(2 \vee 3)}(P)= \begin{cases}A_{1}^{(2)} P^{\beta_{12}} & , P<p_{1}^{(2)} \\ \Phi_{1}^{(2)}(P)+F_{0}^{(2)}(P) & , p_{1}^{(2)} \leq P<\widehat{p}_{1}^{(2)} \\ G_{1}^{(2)} P^{\beta_{22}}+H_{1}^{(2)} P^{\beta_{12}} & , \widehat{p}_{1}^{(2)} \leq P<\widehat{p}_{1}^{(3)} \\ \Phi_{1}^{(3)}(P) & , \widehat{p}_{1}^{(3)} \leq P\end{cases}
$$


The endogenous constants $G_{1}^{(2)}$ and $H_{1}^{(2)}$, as well as the investment thresholds $\widehat{p}_{1}^{(2)}$ and $\widehat{p}_{1}^{(3)}$ are determined via value-matching and smooth-pasting conditions between the three bottom branches of (22). Notice that, in the presence of two market regimes, there exist two waiting regions, i.e., $\left[0, p_{1}^{(2)}\right]$ and $\left[\widehat{p}_{1}^{(2)}, \widehat{p}_{1}^{(3)}\right]$. If $P \in\left[0, p_{1}^{(2)}\right]$, then it is optimal to wait until $P=p_{1}^{(2)}$ and then invest in the second regime holding the option to invest in the third one. However, if $P \in\left[\widehat{p}_{1}^{(2)}, \widehat{p}_{1}^{(3)}\right]$, then it is optimal to invest in either the second regime if $P \downarrow \widehat{p}_{1}^{(2)}$ or the third regime if $P \uparrow \widehat{p}_{1}^{(3)}$. As we will show numerically, greater $\gamma$ or $\sigma_{2}$ reduce the likelihood of direct investment in the second regime by decreasing $\widehat{p}_{1}^{(2)}$, thereby narrowing the wedge between $p_{1}^{(2)}$ and $\widehat{p}_{1}^{(2)}$. This implies that lower risk aversion or higher price uncertainty in the second regime reduce the feasibility of a laggard strategy.

Next, we step back and assume that the third regime is not available yet and that $P<p_{1}^{(1)}$, so that investment in the first regime must be deferred. The dynamies of the yalue function in the first regime under a leapfrog/laggard strategy are described in (23). Notice that, while waiting to invest in the first regime, either an innovation will occur with probability $\nu d t$ and the decisionmaker will receive the value function $F_{1}^{(2 \vee 3)}(P)$, or no innovation will take place with probability $1-\nu d t$ and the decisionmaker will continue holding the value function $\widehat{F}_{1}^{(1)}(P)$.

$$
\widehat{F}_{1}^{(1)}(P)=(1-\rho d t)\left[\nu d t \mathbb{E}\left[F_{1}^{(2 \vee 3)}(P+d P) \mid \mathcal{F}_{0}\right]+(1-\nu d t) \mathbb{E}\left[\widehat{F}_{1}^{(1)}(P+d P) \mid \mathcal{F}_{0}\right]\right]
$$

By expanding the right-hand side of (23) using Itô's lemma we obtain (24), which must be solved for each expression of $F_{1}^{(2 \vee 3)}(P)$ that is indicated in (22).

$$
\frac{1}{2} \sigma_{1}^{2} P^{2} \widehat{F}_{1}^{(1)^{\prime \prime}}(P)+\mu_{1} P \widehat{F}_{1}^{(1)^{\prime}}(P)-(\rho+\nu) \widehat{F}_{1}^{(1)}(P)+\nu F_{1}^{(2 \vee 3)}(P)=0
$$

Solving (24), we obtain the expression for the value function $\widehat{F}_{1}^{(1)}(P)$, which is indicated in $(25)$.

$$
\widehat{F}^{(1)}(P)= \begin{cases}\widehat{A}_{1}^{(2)} P^{\beta_{12}}+E_{1}^{(1)} P^{\delta_{1}} & , P<p_{1}^{(2)} \\ \nu \mathcal{D} \mathcal{A}^{(2)} U\left(P D_{2}\right)-\frac{\nu U\left(c+r I_{1}^{(2)}\right)}{\rho(\rho+\nu)}+B_{0}^{(1)} P^{\beta_{22}} & \\ +K_{1}^{(1)} P^{\delta_{1}}+L_{1}^{(1)} P^{\delta_{2}} & , p_{1}^{(2)} \leq P<\widehat{p}_{1}^{(2)} \\ \widehat{G}_{1}^{(2)} P^{\beta_{22}}+\widehat{H}_{1}^{(2)} P^{\beta_{21}}+Q_{1}^{(1)} P^{\delta_{1}}+R_{1}^{(1)} P^{\delta_{2}} & , \widehat{p}_{1}^{(2)} \leq P<\widehat{p}_{1}^{(3)} \\ \nu \mathcal{D} \mathcal{A}^{(3)} U\left(P D_{3}\right)-\frac{\nu U\left(r I_{1}^{(3)}\right)}{\rho(\rho+\nu)}+J_{1}^{(1)} P^{\delta_{2}} & , \widehat{p}_{1}^{(3)} \leq P\end{cases}
$$

The endogenous constants $E_{1}^{(1)}, K_{1}^{(1)}, L_{1}^{(1)}, Q_{1}^{(1)}, R_{1}^{(1)}$, and $J_{1}^{(1)}$ are obtained analytically via the value-matching and smooth-pasting conditions between the four branches of $(25)$, while $\widehat{A}_{1}^{(2)}, \widehat{G}_{1}^{(2)}$, 
and $\widehat{H}_{1}^{(2)}$ are indicated in (26), (27), and (28), respectively.

$$
\begin{aligned}
& \widehat{A}_{1}^{(1)}=-\frac{\nu A_{1}^{(2)}}{\frac{1}{2} \sigma_{1}^{2} \beta_{12}\left(\beta_{12}-1\right)+\mu_{1} \beta_{12}-(\rho+\nu)} \\
& \widehat{G}_{1}^{(2)}=-\frac{\nu G_{1}^{(2)}}{\frac{1}{2} \sigma_{1}^{2} \beta_{22}\left(\beta_{22}-1\right)+\mu_{1} \beta_{22}-(\rho+\nu)} \\
& \widehat{H}_{1}^{(2)}=-\frac{\nu H_{1}^{(2)}}{\frac{1}{2} \sigma_{1}^{2} \beta_{12}\left(\beta_{12}-1\right)+\mu_{1} \beta_{12}-(\rho+\nu)}
\end{aligned}
$$

Having determined the value of the option to invest under compulsive and leapfrog/laggard strategies, we can compare the value functions $F_{1}^{(1)}(P)$ and $\widehat{F}_{1}^{(1)}(P)$, and, thus, determine the optimal regime-switching strategy endogenously. This is presented in the next section.

\section{Comparison of Regime-Switching Strategies}

Here, we assume that the regime-switching strategy depends on $P, \nu$, and $\gamma$, rather than being determined exogenously by the decisionmaker. Consequently, both compulsive and leapfrog/laggard strategies are possible and the optimal regime-switching strategy is determined endogenously. Figure 2 summarises the optimal strategy for different values of $P, \nu$, and $\gamma$. More specifically, the shaded surface indicates the rate of innovation above which the leapfrog/laggard strategy dominates. Notice that, for all values of $\gamma$ and $\nu$, the compulsive strategy dominates when the output price is low, i.e., $P<\widehat{p}_{1}^{(2)}$. By contrast, the leapfrog/laggard strategy may dominate if $P>\widehat{p}_{1}^{(2)}$. In fact, risk-seeking behaviour $(\lambda>1)$ raises the likelihood of a leapfrog/laggard strategy by lowering the required rate of innovation above which this strategy is optimal. Interestingly, a leapfrog/laggard strategy may dominate even if the decisionmaker is risk averse $(\gamma<1)$, provided, however, that both the rate of innovation and the output price are sufficiently high.

In more detail, the compulsive strategy always dominates when the output price is low because, even if a new market regime was available, the decisionmaker would still have to wait long before the output price reaches the corresponding investment threshold. Consequently, the expected payoff from investment in the new regime does not offset the forgone revenues from skipping the old one. This has been shown by Chronopoulos and Siddiqui (2015) under risk neutrality, i.e., $\gamma=1$, and Proposition 7 extends this result to the case $\gamma \in[0.5,1.5]$.

Proposition 7. If $P<\widehat{p}_{1}^{(2)}$, then $F_{1}^{(1)}>\widehat{F}_{1}^{(1)} \forall \gamma \in[0.5,1.5]$ and $\forall \nu \in[0,1]$.

Interestingly, however, even when the decisionmaker is risk averse, a high output price combined with a high rate of innovation may increase the incentive to delay investment until a new market regime emerges before deciding which regime to invest in. More specifically, as Proposition 8 


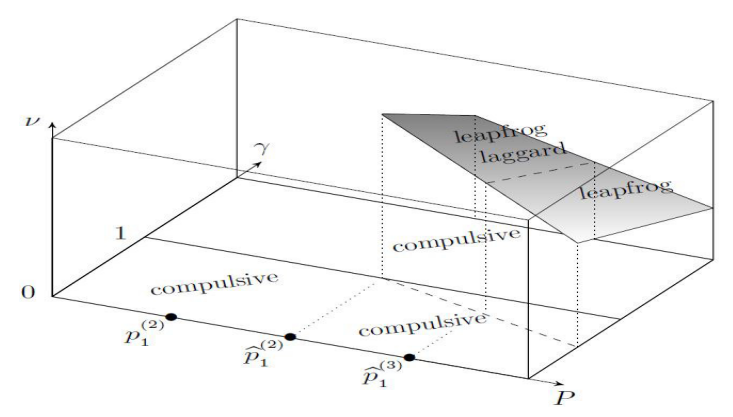

Figure 2: Comparison of the strategies

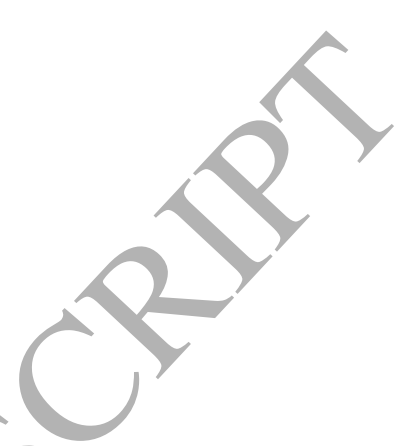

indicates, for each output price $P>\widehat{p}_{1}^{(1)}$ there exists a set $\mathbb{G}_{n} \subset[0.5,1], n \in \mathbb{N}$ of values of $\gamma$, such that the leapfrog/laggard strategy dominates provided that the rate of innovation is sufficiently high. In fact, $\mathbb{G}_{n}$ represents a family of increasing subsets such that $\mathbb{G}_{n-1} \subset \mathbb{G}_{n} \subset[0.5,1]$. Nevertheless, for a given output price $P>\widehat{p}_{1}^{(1)}$, greater risk aversion (lower $\gamma$ ) raises the required rate of innovation for which the decisionmaker is willing to consider a leapfrog/laggard strategy. By contrast, a ceteris paribus increase in $\gamma$ lowers the required rate $\nu$ for which the leapfrog/laggard strategy dominates. In turn, this implies that as the decisionmaker becomes less risk averse, she is more willing to tolerate a lower rate of innovation in order to wait for the next regime to appear before considering which regime to invest in.

Proposition 8. $\forall P>\widehat{p}_{1}^{(2)}, \exists \mathbb{G}_{n} \neq \emptyset, n \in \mathbb{N}$ with $\mathbb{G}_{n-1} \subset \mathbb{G}_{n} \subset[0.5,1]: \forall \gamma \in \mathbb{G}_{n}, \exists \bar{\nu} \in[0,1]:$ $\widehat{F}_{1}^{(1)} \geq F_{1}^{(1)}, \forall \nu \geq \bar{\nu}$

Furthermore, as we will illustrate numerically, an increase in price uncertainty in the second regime decreases the feasibility of the laggard strategy by narrowing the wedge between $p_{1}^{(2)}$ and $\widehat{p}_{1}^{(2)}$, and, in turn, the range of output prices in which immediate investment in the second regime is possible. The same effect is observed with lower risk aversion, as it reduces both $p_{1}^{(2)}$ and $\widehat{p}_{1}^{(2)}$, yet has a more pronounced effect on the latter. This result is in line with Proposition 8, as it implies that lower risk aversion increases the attractiveness of a leapfrog strategy. By contrast, an increase in price uncertainty in the third (new) regime raises both $\widehat{p}_{1}^{(2)}$ and $\widehat{p}_{1}^{(3)}$, yet has a more pronounced effect on the latter. In turn, this implies that greater price uncertainty in the new market regime increases the wedge between $\widehat{p}_{1}^{(2)}$ and $\widehat{p}_{1}^{(3)}$. Consequently, while greater price uncertainty in the third regime raises the incentive to abandon the second one under a compulsive strategy, it increases the feasibility of the laggard strategy when the decisionmaker has yet to invest in the latter regime. 


\section{Numerical Examples}

For the numerical examples we assume that $\mu_{1}=0.01, \mu_{2}=0.001, \mu_{3}=0.015$ and that $\sigma_{k} \in$ $[0,0.2], k=1,2,3$. Also, unless stated otherwise, $D_{1}=1, D_{2}=0.7$, and $D_{3}=2$, while $I_{1}^{(1)} \equiv I_{1}^{(2)}=$ $100, I_{0}^{(2)}=50$, and $I_{1}^{(3)}=1000$. The operating cost is $c=10$ and $\nu \in[0,1]$. Consequently, the assumptions $\mu_{3}>\mu_{1}>\mu_{2}$ and $\frac{D_{3}}{I_{1}^{(3)}}<\frac{D_{2}}{I_{1}^{(2)}}$ are satisfied. The left panel in Figure 3 illustrates the impact of $\gamma$ on the option and project value in the third regime, while, the right panel, illustrates the impact of $\gamma$ on the required investment threshold, $p_{1}^{(3)}$, for $\sigma_{3}=0.15,0.17$, and 0.2 . Note that $\gamma<1(\gamma>1)$ implies that the decisionmaker is risk averse (risk seeking) and that the expected utility of the project is concave (convex). Consequently, an increase (decrease) in $\gamma$ raises (lowers) the expected utility of the active project and lowers (raises) the required investment threshold. This is also illustrated in the right panel, which indicates that an increase in price uncertainty raises the required investment threshold by increasing the value of waiting.
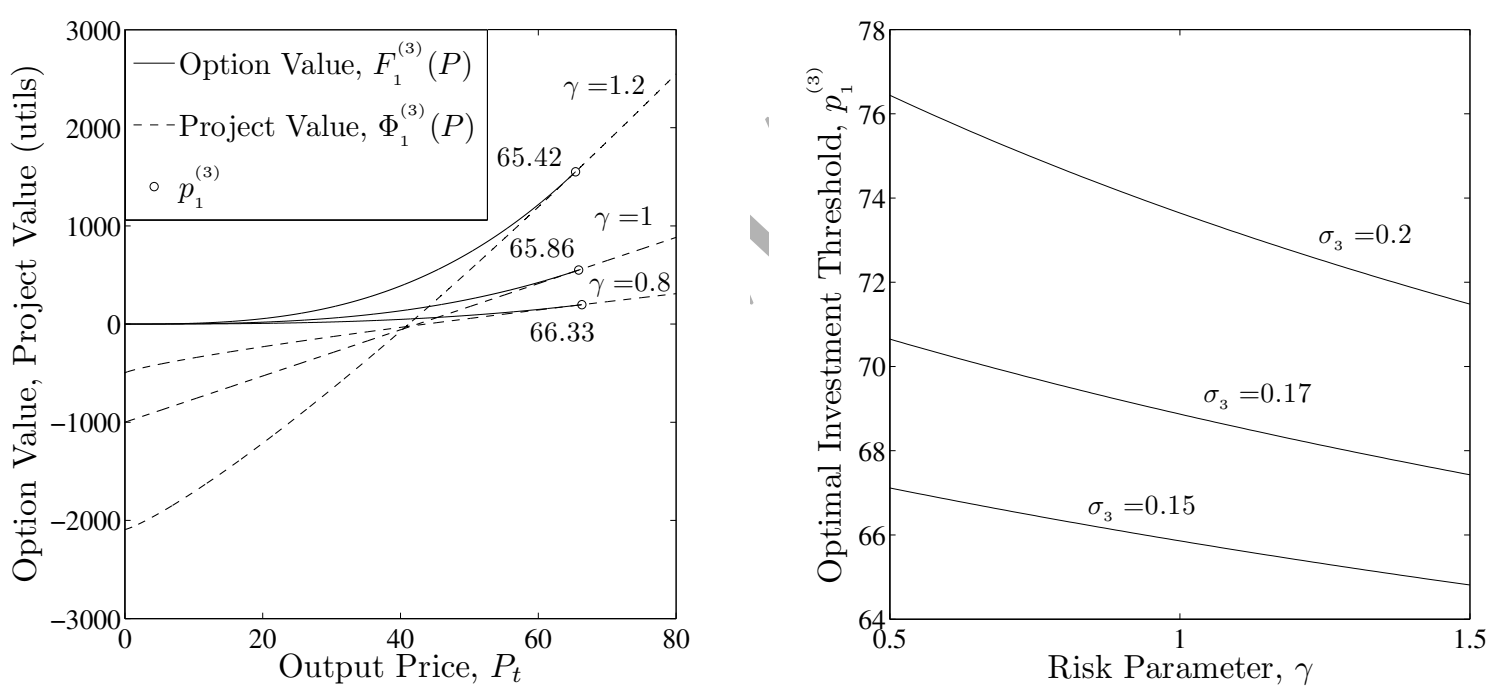

Figure 3: Impact of $\gamma$ on option and project value for $\sigma_{3}=0.15$ and $D_{3}=2$ (left) and $p_{1}^{(3)}$ versus $\gamma$ and $\sigma$ (right)

Figure 4 illustrates the impact of $\gamma$ on the required abandonment threshold for $\sigma_{2}=0.2$ and $\sigma_{3}=$ 0.15 (left panel) and $\sigma_{2}, \sigma_{3}=0.15,0.2$ (right panel). According to both panels, the option to invest in the third regime increases the value of the option to abandon the second one, thereby raising the required abandonment threshold. Additionally, the impact of risk aversion on the decision to abandon the second regime is non-monotonic and this effect is more pronounced as market regimes become more asymmetric. More specifically, greater risk aversion, i.e., lower $\gamma$, accelerates abandonment, whereas, if the decisionmaker is risk seeking, then greater $\gamma$ delays abandonment when $\gamma$ is small and facilitates abandonment when $\gamma$ is large. In fact, this result becomes more pronounced as the market share of the third regime increases (left panel). Intuitively, a ceteris 
paribus increase in $D_{3}$ raises the attractiveness of the third regime, and, in turn, the incentive to abandon the second one. This is in contrast to the symmetric framework of Chronopoulos et al. (2011), who show how greater risk aversion postpones the suspension of a project prior to permanent resumption. More specifically, they attribute the impact of $\gamma$ on the required suspension threshold to the reluctance of the decisionmaker to suspend operations in view of facing lower revenues upon resumption.

By contrast, the current model differs not only by relaxing the assumption of costless abandonment but also with respect to the asymmetries of the different market regimes. Consequently, the numerical results within an asymmetric framework imply that the impact of $\gamma$ on the decision to abandon the second regime can be explained by the particular characteristics of the different market regimes. As the right panel illustrates, greater price uncertainty in the second regime lowers the required abandonment threshold. This happens because the decisionmaker has a lower incentive to abandon the project in case of a temporary downturn, which is more likely when uncertainty is high. However, a ceteris paribus increase in uncertainty in the third regime accelerates abandonment of second one. Intuitively, greater uncertainty in the third regime raises the value of the corresponding investment opportunity, thereby increasing the incentive to abandon the second regime. This is contrary to the standard real options intuition, according to which greater uncertainty postpones abandonment by increasing the value of waiting. Notice also that this result holds under both risk-averse and risk-seeking behaviour, yet it is more pronounced in the latter case.
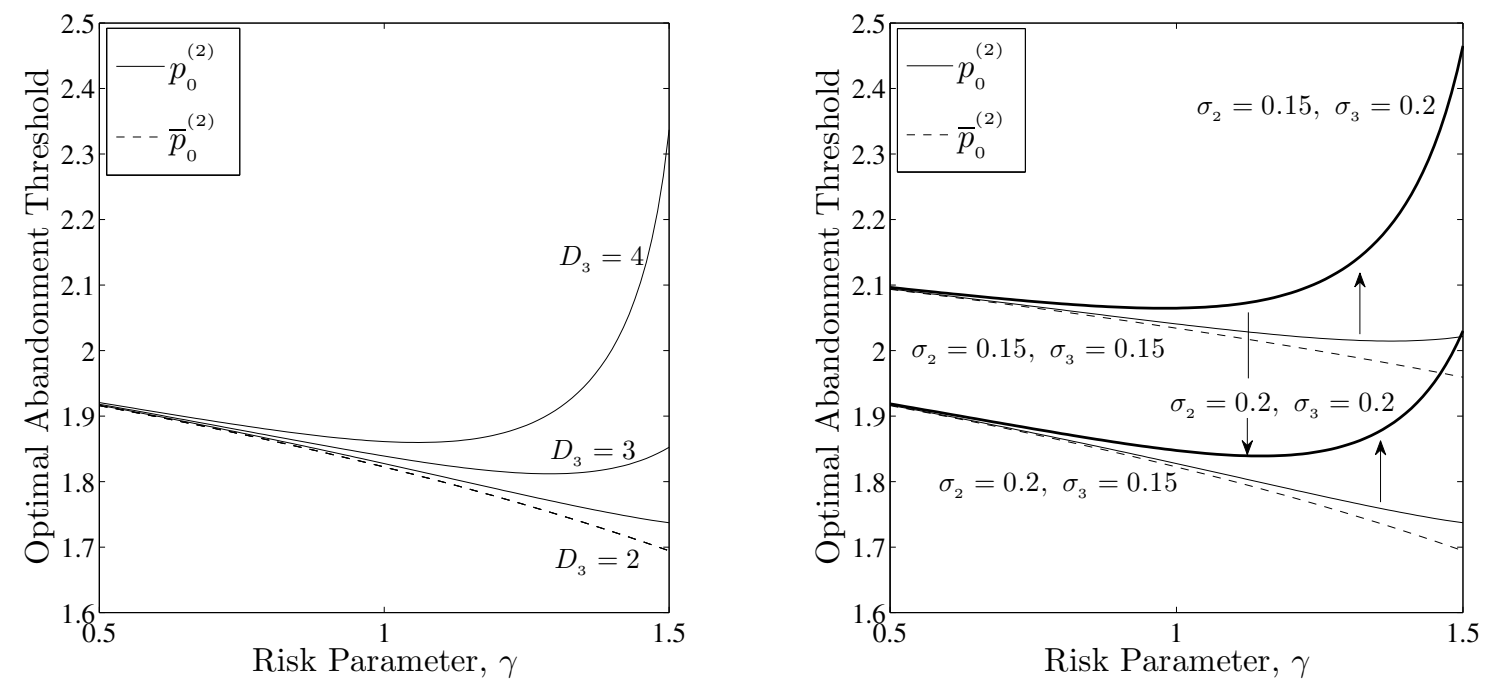

Figure 4: Optimal abandonment threshold, $p_{0}^{(2)}$ and $\bar{p}_{0}^{(2)}$, versus $\gamma$ for $\sigma_{2}=0.2$ and $\sigma_{3}=0.15$ (left) and $\sigma_{2}, \sigma_{3}=$ $0.15,0.2$ (right)

The left panel in Figure 5 illustrates how the likelihood of regime switching impacts the value of the investment opportunity and the value of the project in the first regime, while the right 
panel illustrates how both the likelihood of regime switching and risk aversion impact the required investment threshold, $p_{1}^{(1)}$. According to the left panel, a greater likelihood of regime switching lowers the expected utility of the project's cash flows, and, in turn, the incentive to invest. As the right panel illustrates, this result is more pronounced under greater risk aversion, which reduces the expected utility of the project, thereby further reducing the investment incentive.
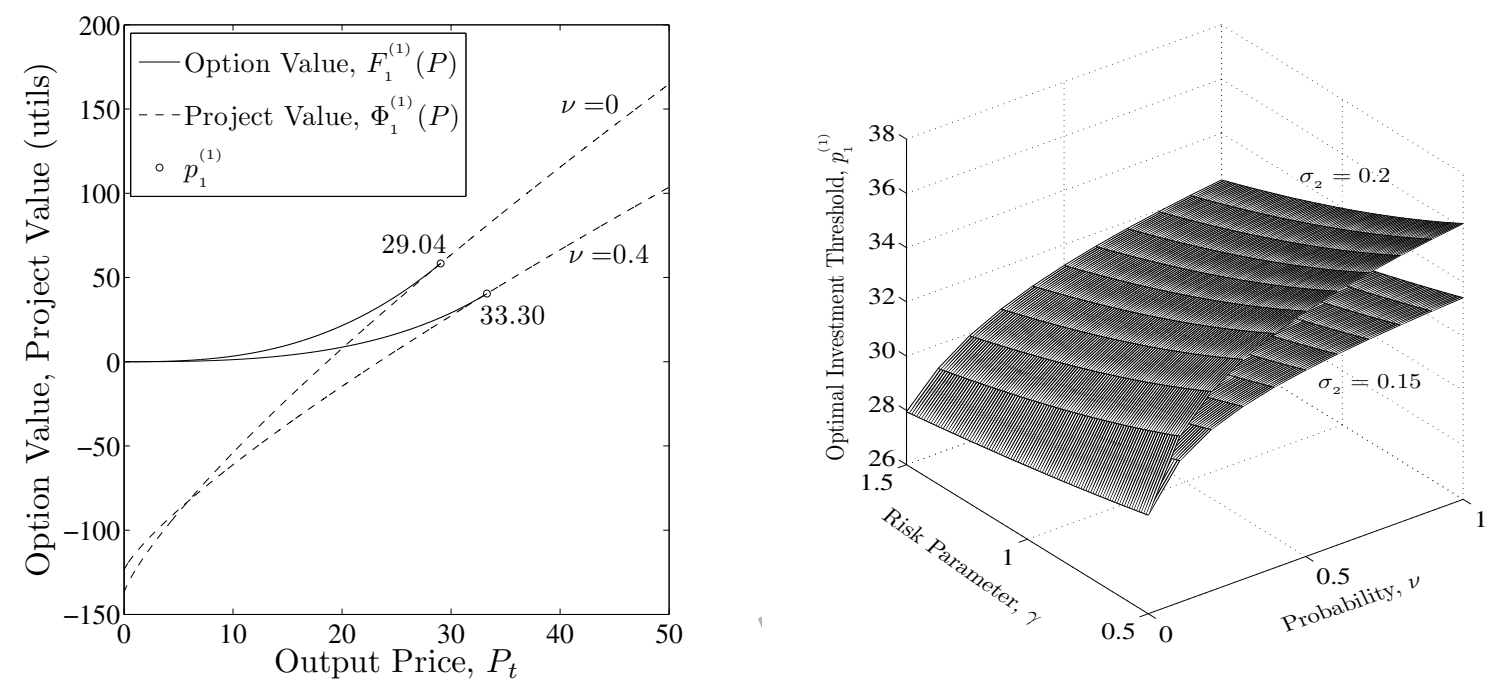

Figure 5: Option and project value versus $\nu$ for $\gamma=0.8$ and $\sigma_{1}=0.18, \sigma_{2}=0.2$, and $\sigma_{3}=0.15$ (left) and optimal investment threshold, $p_{1}^{(1)}$, versus $\nu$ and $\gamma$ for $\sigma_{1}=0.18, \sigma_{2}=0.15,0.2$, and $\sigma_{3}=0.15$ (right)

The left panel in Figure 6 illustrates the value of the option to choose between the second and the third market regime, as well as the value function in the first regime under a leapfrog/laggard strategy for $\gamma=0.6,0.8$. Notice that the presence of two market regimes creates two waiting regions. In the first waiting region, the output price is very low, and, therefore, it is optimal to wait until $P=p_{1}^{(2)}$ and then invest in the second regime. For $\gamma=0.6$, the first waiting region is $[0,39.1]$, and, thus, $p_{1}^{(2)}=39.1$. The second waiting region corresponds to an area around the indifference point between the two regimes, which is located at the intersection between the two NPVs. For $\gamma=0.6$, the second waiting region is [59.95, 76.52]. If $P \in[59.95,76.52]$, then it is optimal to invest in either the second regime if $P \downarrow 59.95$ or the third regime if $P \uparrow 76.52$. Additionally, a higher rate of innovation raises the likelihood of the arrival of a new market regime, and, in turn, the value function in the first regime, so that $\nu \rightarrow \infty \Rightarrow \widehat{F}_{1}^{(1)}(P) \rightarrow F_{1}^{(2 \vee 3)}(P)$. Notice that lower risk aversion narrows the interval $\left[p_{1}^{(2)}, \widehat{p}_{1}^{(2)}\right]$, thereby reducing the range of values of the output price for which a laggard strategy is feasible, and, in turn, promoting a leapfrog strategy. The right panel illustrates the impact of higher $\sigma_{2}$ and $\sigma_{3}$ on the required investment thresholds $p_{1}^{(2)}, \widehat{p}_{1}^{(2)}$, and $\widehat{p}_{1}^{(3)}$. Although lower risk aversion reduces the feasibility of the laggard strategy, greater price uncertainty in the 
third regime, which is indicated by the direction of the arrows, increases the range of values of $\sigma_{2}$ for which direct investment in the second regime is possible. By contrast, greater price uncertainty in the second regime narrows the range of prices for which direct investment in the second regime is possible. Hence, a laggard strategy becomes less (more) feasible under greater price uncertainty in the second (third) regime. Intuitively, the impact of price uncertainty in the second regime becomes relatively less pronounced when price uncertainty in the third regime increases.
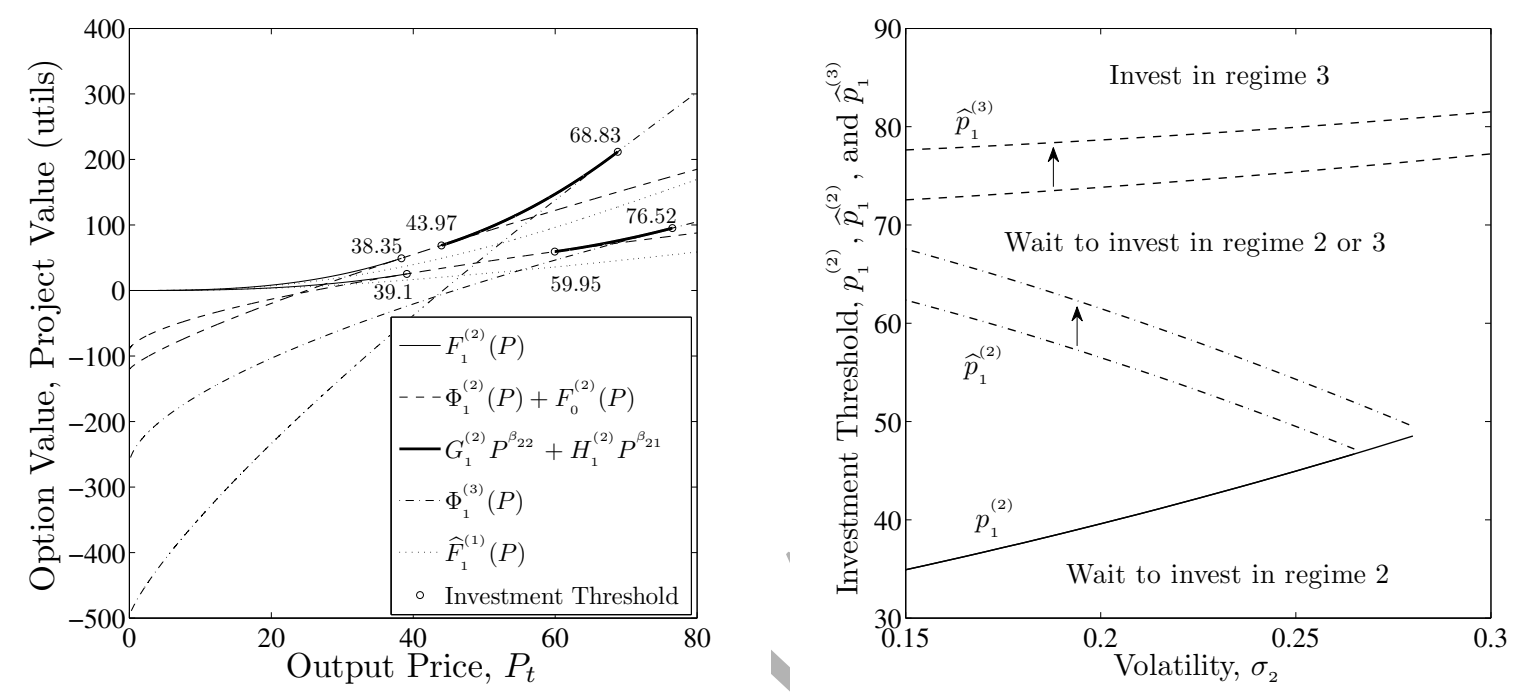

Figure 6: Choosing between alternative market regimes for $\gamma=0.6,0.8, \sigma_{2}=0.2$, and $\sigma_{3}=0.15$ (left) and optimal investment thresholds, $p_{1}^{(2)}, \widehat{p}_{1}^{(2)}$, and $\widehat{p}_{1}^{(3)}$, versus $\sigma_{2}$ for $\gamma=0.6$ and $\sigma_{3}=0.15,0.2$ (right)

Figure 7 illustrates the relative value of the two strategies, i.e., compulsive and leapfrog/laggard, under a low (left panel) and high output price (right panel) for different levels of $\gamma$ and $\nu$. The relative value of the two strategies for $P<p_{1}^{(2)}$ is indicated in (29) and is always greater than one, as the left panel illustrates. This implies that, if the output price is low, then the compulsive strategy dominates the leapfrog/laggard strategy under both risk-averse and risk-seeking behaviour. This happens because the decisionmaker would have to wait long before investment in the third regime is justified and the expected revenues from investing in the third regime do not compensate the forgone revenues from ignoring the second one.

$$
\frac{A_{1}^{(1)} P^{\delta_{1}}+C_{1}^{(1)} P^{\beta_{21}}}{\widehat{A}_{1}^{(2)} P^{\beta_{12}}+E_{1}^{(1)} P^{\delta_{1}}}
$$

The relative value of the two strategies for $P \in\left[\widehat{p}_{1}^{(2)}, \widehat{p}_{1}^{(3)}\right]$ and $P \in\left[\widehat{p}_{1}^{(3)}, \infty\right)$ is indicated in (30). As the right panel illustrates, the relative value of the two strategies may drop below one even when the decisionmaker is risk averse provided that both the output price and the rate of innovation are high. More specifically, if $P \in\left[\widehat{p}_{1}^{(2)}, \widehat{p}_{1}^{(3)}\right]$, then the relative value of the two strategies becomes less 
than one only under risk-seeking behaviour. By contrast, if $P\left[\widehat{p}_{1}^{(3)}, \infty\right)$, then the relative value of the two strategies may drop below one even when the decisionmaker is risk averse. This implies that although risk aversion typically promotes a compulsive strategy by decreasing the expected utility of the project, a higher output price or innovation rate mitigate this effect, thereby increasing the incentive to adopt a leapfrog/laggard strategy.

$$
\frac{\Phi_{1}^{(1)}(P)}{\widehat{G}_{1}^{(2)} P^{\beta_{22}}+\widehat{H}_{1}^{(2)} P^{\beta_{21}}+Q_{1}^{(1)} P^{\delta_{1}}+R_{1}^{(1)} P^{\delta_{2}}} \quad \text { and } \frac{\Phi_{1}^{(1)}(P)}{\nu \mathcal{D} \mathcal{A}^{(3)} U\left(P D_{3}\right)-\frac{\nu}{\rho+\nu} U\left(r I_{1}^{(3)}\right)+J_{1}^{(1)} P^{\delta_{2}}}
$$
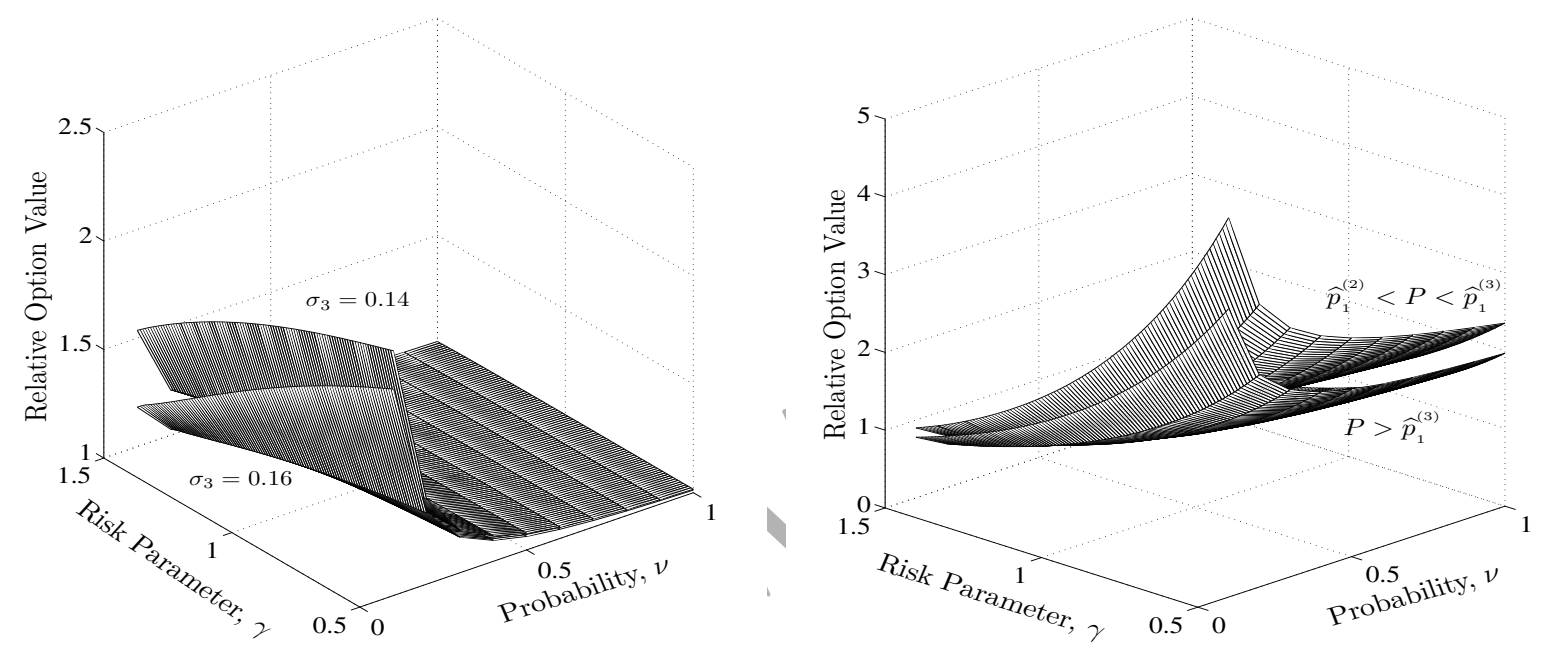

Figure 7: Relative value of the two strategies under a low $0<P<p_{1}^{(2)}$ (left) and high output price $\widehat{p}_{1}^{(2)} \leq P<\widehat{p}_{1}^{(3)}$ and $\widehat{p}_{1}^{(3)} \leq P$ (right)

\section{Conclusions}

Failure to recognise the developing world as a rapid-growing market and not just a low-cost manufacturing base, can impact the viability of firms investing in emerging markets significantly. Therefore, we develop a utility-based framework with regime switching in order to analyse how attitudes towards risk affect incentives for technological change. Assuming that the arrival of innovations follows a Poisson process and that price uncertainty is modelled via a Markov-modulated GBM, we analyse thee different regime-switching strategies, namely compulsive, leapfrog, and laggard. In the first strategy, the decisionmaker invests in each market regime that becomes available, whereas in the second and third ones, the decisionmaker first waits for a new market regime to emerge and then decides whether to invest in an older or a newer regime, respectively.

Results indicate that attitudes towards risk and the relative characteristics of each market regime can have crucial implications for the optimal regime-switching strategy. More specifically, 
while we confirm that greater risk aversion delays investment by decreasing the expected utility of a project (Hugonnier \& Morellec, 2013), we also show that if market regimes are asymmetric, then greater risk aversion has a non-monotonic impact on the decision to abandon an old market regime and may either increase or decrease the required abandonment threshold. This is in contrast to Chronopoulos et al. (2011), who show that greater risk aversion delays the temporary suspension of a project prior to permanent resumption. However, the latter assume that there is no cost associated with operational decisions and that market regimes are symmetric, since the decisionmaker resumes the same project after temporary suspension.

By contrast, in this paper we assume that, after abandoning an old market regime, the decisionmaker has the option to enter a new one that covers a greater market share. In addition, we assume that entry into the new market regime entails a much greater investment cost. Under these assumptions, we find that the incentive to abandon an old regime is greater as the market share of the new regime increases. Interestingly, the incentive to abandon an old regime in order to enter a new one is greater when price uncertainty in the new regime increases, as this raises the value of the corresponding investment opportunity. Also, we show that, although a compulsive strategy always dominates when the output price is low, a leapfrog/laggard strategy may dominate even when the decisionmaker is risk averse, provided that both the output price and the rate of innovation are sufficiently high. This has crucial implications for investment in emerging markets, as it implies that entry in a new and potential more risky market regime is not necessarily associated with risk-seeking behaviour, and may be the optimal investment strategy even when decisionmakers are risk averse.

The model presented in this paper may also accommodate other aspects of the real options literature, thereby offering a flexible framework for several meaningful extensions of existing real options models. For example, directions for further research may include the analysis of other forms of managerial discretion, such as discretion over project scale, thus extending Dangl (1999) and Hagspiel et al. (2013). Additionally, strategic interactions may also be considered by extending the current framework to allow for duopolistic competition, as in Goto et al. (2013). Furthermore, the application of an alternative stochastic process such as an arithmetic Brownian motion could provide information regarding the robustness of the numerical, theoretical, and intuitive results. Also, a different class of utility functions could be applied in order to obtain further insight regarding the impact of risk aversion on the optimal investment policy and allow for comparisons with the approach presented in this paper. Finally, a meaningful extention in the same line of work would be to study how the discount rate may be affected by a decisionmaker's attitudes towards risk. 


\section{APPENDIX}

\section{Regime 3}

Proposition 1 The objective function $F_{1}^{(3)}(P)$ is strictly concave at $p_{1}^{(3)}$.

Proof: The objective function can be expressed as in (B-1).

$$
F_{1}^{(3)}(P)=\left(\frac{P}{p_{1}^{(3)}}\right)^{\beta_{13}}\left[\mathcal{A}^{(3)} U\left(p_{1}^{(3)} D_{3}\right)-U\left(r I_{1}^{(3)}\right)\right]
$$

Differentiating (B-1) twice with respect to $p_{1}^{(3)}$, we can express the SOSC as in (B-2).

$$
\frac{1}{p_{1}^{(3)^{2}}}\left[\left(\beta_{13}+\beta_{13}^{2}\right)\left[\mathcal{A}^{(3)} U\left(p_{1}^{(3)} D_{3}\right)-U\left(r I_{1}^{(3)}\right)\right]+\mathcal{A}^{(3)} U\left(p_{1}^{(3)} D_{3}\right)\left(\gamma-1-2 \beta_{13}\right)\right]<0
$$

By substituting the expression for $p_{1}^{(3)}$ into (B-2), we obtain (B-3).

$$
\begin{aligned}
& \frac{\beta_{13} \beta_{23}}{\left(\gamma-\beta_{13}\right)\left(\gamma-\beta_{23}\right)} \frac{\beta_{23}-\gamma}{\beta_{23}}\left[\beta_{13}\left(\beta_{13}+1\right)+\gamma^{2}-\gamma-2 \beta_{13} \gamma\right]-\beta_{13}\left(\beta_{13}+1\right)<0 \\
\Leftrightarrow & \frac{1}{\beta_{13}-\gamma}\left[\beta_{13}\left(\beta_{13}+1\right)+\gamma^{2}-\gamma-2 \beta_{13} \gamma\right]-\left(\beta_{13}+1\right)<0 \Leftrightarrow \gamma>0
\end{aligned}
$$

The last inequality is true since since $\gamma \in[0.5,1.5]$.

Proposition $2 \forall \sigma_{3}>0$ and $\forall \gamma \in[0.5,1.5]$ we have $\frac{\partial p_{1}^{(3)}}{\partial \sigma_{3}}>0$ and $\frac{\partial p_{1}^{(3)}}{\partial \gamma}<0$.

Proof: Differentiating $p_{1}^{(3)}$ with respect to $\sigma_{3}$ we have:

$$
\frac{\partial p_{1}^{(3)}}{\partial \sigma_{3}}=\frac{r I_{1}^{(3)}}{D_{3}}\left(\frac{\beta_{23}-\gamma}{\beta_{23}}\right)^{\frac{1-\gamma}{\gamma}} \frac{1}{\beta_{23}^{2}} \frac{\partial \beta_{23}}{\partial \sigma_{3}}
$$

Since $\frac{\partial \beta_{23}}{\partial \sigma_{3}}>0$, we have $\frac{\partial p_{1}^{(3)}}{\partial \sigma_{3}}>0$. Next, we differentiate $p_{1}^{(3)}$ with respect to $\gamma$.

$$
\frac{\partial p_{1}^{(3)}}{\partial \gamma}<0 \Leftrightarrow \frac{\partial}{\partial \gamma}\left[\ln \frac{r I_{1}^{(3)}}{D_{3}}+\frac{1}{\gamma} \ln \left(\frac{\beta_{23}-\gamma}{\beta_{23}}\right)\right]<0 \Leftrightarrow \ln \left(\frac{\beta_{23}-\gamma}{\beta_{23}}\right)>1-\frac{\beta_{23}}{\beta_{23}-\gamma}
$$

By setting $x=\frac{\beta_{23}}{\beta_{23}-\gamma}$, (B-5) can be written as $\ln x<x-1$. Note that in order to show the latter, we first need to show that $e^{x} \geq 1+x \forall x$. Therefore, we assume a $\lambda \in \mathbb{N}$ such that $\lambda>-x$ or equivalently $\lambda-x \geq 0, \forall x \in \mathbb{R}$. This implies that $1+\frac{x}{\lambda} \geq 0$ and from Bernoulli's inequality we have $\left(1+\frac{x}{\lambda}\right)^{\lambda} \geq 1+\lambda \frac{x}{\lambda}=1+x$. Consequently:

$$
e^{x}=\lim _{\lambda \rightarrow \infty}\left(1+\frac{x}{\lambda}\right)^{\lambda} \geq \lim _{\lambda \rightarrow \infty}(1+x) \Rightarrow e^{x} \geq 1+x, \forall x \in \mathbb{R}
$$

Hence, assuming that $x>0$ and setting $\ln x$ instead of $x$ we finally have $e^{\ln x}=x \geq 1+\ln x \Rightarrow$ $\ln x \leq x-1$. 


\section{Regime 2}

Proposition 3 The objective function $\bar{F}_{0}^{(2)}(P)$ is strictly concave at $\bar{p}_{0}^{(2)}$.

Proof: The derivation follows the same steps as in Proposition 1.

Proposition $4 \forall \sigma_{2}>0$ and $\forall \gamma \in[0.5,1.5]$ we have $\frac{\partial \bar{p}_{0}^{(2)}}{\partial \sigma_{2}}<0$ and $\frac{\partial \bar{p}_{0}^{(2)}}{\partial \gamma}<0$.

Proof: The derivation follows the same steps as in Proposition 2.

Proposition 5 A ceteris paribus increase in $D_{3}$ raises $p_{0}^{(2)}$.

Proof: First, we rewrite the FONC for the optimal abandonment threshold $p_{0}^{(2)}$ in (B-7) by equating the MB (left-hand side) of accelerating abandonment to the MC (right-hand side).

$$
\begin{aligned}
& \left(\beta_{13}-\beta_{22}\right)\left(\frac{p_{0}^{(2)}}{p_{1}^{(3)}}\right)^{\beta_{13}} \mathcal{A}^{(3)} U\left(p_{1}^{(3)} D_{3}\right)-\frac{\beta_{22}}{\rho} U\left(c D_{2}-r I_{0}^{(2)}\right) \\
& =\gamma \mathcal{A}^{(2)} U\left(p_{0}^{(2)} D_{2}\right)-\beta_{22} \mathcal{A}^{(2)} U\left(p_{0}^{(2)} D_{2}\right)+\left(\beta_{13}-\beta_{22}\right)\left(\frac{p_{0}^{(2)}}{p_{1}^{(3)}}\right)^{\beta_{13}} \frac{U\left(r I_{1}^{(3)}\right)}{\rho}
\end{aligned}
$$

Notice that an increase in $D_{3}$ raises the $\mathrm{MB}$ of accelerating abandonment without affecting the MC. Consequently, the marginal utility of accelerating abandonment increases, thereby raising the incentive to abandon the second regime.

Proposition 6 A ceteris paribus increase in $\sigma_{3}\left(\sigma_{2}\right)$ increases (decreases) $p_{0}^{(2)}$.

Proof: From (8) we know that an increase in $\sigma_{2}$ does not impact the decision to invest in the third regime. Consequently, the impact of $\sigma_{2}$ on $p_{0}^{(2)}$ is the same as that on $\bar{p}_{0}^{(2)}$, and, from Proposition 4, we conclude that $\frac{\partial p_{0}^{(2)}}{\partial \sigma_{2}}<0$. Also, the impact of $\sigma_{3}$ is isolated on the first and third term on left- and right-hand side of (B-7), respectively. Hence, the result follows if we show that the MB of accelerating abahdonment increases by more than the MC.

Notice that the impact of $\sigma_{3}$ on $\left(\beta_{13}-\beta_{22}\right)\left(\frac{p_{0}^{(2)}}{p_{1}^{(3)}}\right)^{\beta_{13}}$ is common for both the left and the righthand side of (B-7), and, therefore, the impact of $\sigma_{3}$ on the MB and MC of accelerating abandonment depends on its impact on $\mathcal{A}^{(3)} U\left(p_{1}^{(3)} D_{3}\right)$. From Proposition 2, we know that $\frac{\partial p_{1}^{(3)}}{\partial \sigma_{3}}>0$, and, therefore, we conclude that $\frac{\partial}{\partial \sigma_{3}} \mathrm{MB}>\frac{\partial}{\partial \sigma_{3}} \mathrm{MC}, \forall \gamma$. Consequently, an increase in uncertainty in the third regime accelerates abandonment of the second one. Note $\frac{\partial \mathcal{A}^{(3)}}{\partial \sigma_{3}}<0$ if $\gamma<1$, whereas $\frac{\partial \mathcal{A}^{(3)}}{\partial \sigma_{3}}>0$ if $\gamma>1$. Consequently, this result is less pronounced under risk aversion $(\gamma<1)$, since the decrease in $\mathcal{A}^{(3)}$ makes the increase in $\mathcal{A}^{(3)} U\left(p_{1}^{(3)} D_{3}\right)$ less pronounced. 


\section{Regime 1}

By expanding the right-hand side of (16) using Itô's lemma, we obtain (B-8).

$$
\begin{aligned}
& \frac{1}{2} \sigma_{1}^{1} P^{2} \Phi_{1}^{(1)^{\prime \prime}}(P)+\mu_{1} P \Phi_{1}^{(1)^{\prime}}(P)-(\rho+\nu) \Phi_{1}^{(1)}(P)+\nu\left[\mathcal{A}^{(2)} U\left(P D_{2}\right)-\frac{1}{\rho} U\left(c D_{2}+r I_{1}^{(1)}\right)\right. \\
& \left.+A_{0}^{(2)} P^{\beta_{22}}\right]+U\left(P D_{1}\right)-U\left(c D_{1}+r I_{1}^{(1)}\right)=0
\end{aligned}
$$

We begin with the non-homogenous differential equation (B-9), for which the particular solution takes the form $\phi_{1}(P)=\mathcal{D} U\left(P D_{1}\right)+\nu \mathcal{D} \mathcal{A}^{(2)} U\left(P D_{2}\right)$.

$$
\frac{1}{2} \sigma_{1}^{1} P^{2} \Phi_{1}^{(1)^{\prime \prime}}(P)+\mu_{1} P \Phi_{1}^{(1)^{\prime}}(P)-(\rho+\nu) \Phi_{1}^{(1)}(P)+U\left(P D_{1}\right)+\nu \mathcal{A}^{(2)} U\left(P D_{2}\right)=0
$$

Next, we conjecture that the particular solution for $(\mathrm{B}-10)$ has the form $\phi_{2}(P)=B_{0}^{(2)} P^{\beta_{22}}$.

$$
\frac{1}{2} \sigma_{1}^{1} P^{2} \Phi_{1}^{(1)^{\prime \prime}}(P)+\mu_{1} P \Phi_{1}^{(1)^{\prime}}(P)-(\rho+\nu) \Phi_{1}^{(1)}(P)+\nu A_{0}^{(2)} P^{\beta_{22}}=0
$$

By substituting $\phi_{2}(P)$ into (B-10) we have $\left[\frac{1}{2} \sigma_{1}^{2} \beta_{22}\left(\beta_{22}-1\right)+\mu_{1} \beta_{22}-(\rho+\nu)\right] B_{0}^{(2)}+\nu A_{0}^{(2)}=0$. Finally, the particular solutions corresponding to the non-stochastic terms in $(\mathrm{B}-8)$ are $\phi_{3}(P)=$ $\frac{\nu}{\rho(\rho+\nu)} U\left(c D_{2}+r I_{1}^{(1)}\right)$ and $\phi_{4}(P)=\frac{1}{\rho+\nu} U\left(c D_{1}+r I_{1}^{(1)}\right)$. Thus, $\Phi_{1}^{(1)}(P)=\sum_{j} \phi_{j}(P)$.

Next, the dynamics of the value function $F_{1}^{(1)}(P)$ are described in (18). By expanding the righthand side of (18) using Itô's lemma we obtain (19), which must be solved together with (20). The solution to the homogenous part of $(18)$ is $A_{1}^{(1)} P^{\delta_{1}}$. In addition, we conjecture that a particular solution for the non-homogenous differential equation (18) is of the form $C_{1}^{(1)} P^{\beta_{21}}$. By substituting the latter into (18), we obtain the expression for $C_{1}^{(1)}$.

Proposition 7 If $P<\widehat{p}_{1}^{(2)}$, then $F_{1}^{(1)}>\widehat{F}_{1}^{(1)} \forall \gamma \in[0.5,1.5]$ and $\forall \nu \in[0,1]$.

Proof: If $\Phi_{1}^{(3)}(\widetilde{p})<0$, where $\widetilde{p}$ is such that $\Phi_{1}^{(2)}(\widetilde{p})=\Phi_{1}^{(3)}(\widetilde{p})$, then the investment region is not dichotomous and it is optimal to wait until $P=\widehat{p}_{1}^{(3)}$ and then invest in the third regime (Dixit, 1993). However, if $\Phi_{1}^{(3)}(\widetilde{p})>0$, then, according to Décamps et al. (2006), the investment region is dichotomous and the expected NPV from investment in the first technology under a compulsive strategy is indicated in (13). Although the payoff under a laggard strategy is the same, it is, nevertheless, conditional on the arrival of the second technology, and, therefore, is lower compared to the immediate profit from a compulsive strategy. Hence, the compulsive strategy always dominates if $P<\widehat{p}_{1}^{(2)}$.

Proposition $8 \forall P>\widehat{p}_{1}^{(2)}, \exists \mathbb{G}_{n} \neq \emptyset, n \in \mathbb{N}$ with $\mathbb{G}_{n-1} \subset \mathbb{G}_{n} \subset[0.5,1]: \forall \gamma \in \mathbb{G}_{n}, \exists \bar{\nu} \in[0,1]$ : $\widehat{F}_{1}^{(1)} \geq F_{1}^{(1)}, \forall \nu \geq \bar{\nu}$. 
Proof: Although the expression for $\widehat{F}_{1}^{(1)}(P)$ is different for $P>\widehat{p}_{1}^{(3)}$ and $\widehat{p}_{1}^{(2)} \leq P \leq \widehat{p}_{1}^{(3)}$, since both $\widehat{F}_{1}^{(1)}(P)$ and $\Phi_{1}^{(1)}(P)$ are $\mathcal{C}^{1}$, we will show the result for $P>\widehat{p}_{1}^{(3)}$. The value function under a leapfrog strategy is indicated in (25), from which we obtain (B-11)

$$
\widehat{F}_{1}^{(1)}(P)= \begin{cases}0 & , \nu=0 \\ \mathcal{A}^{(3)} U\left(P D_{3}\right)-\frac{1}{\rho} U\left(r I_{1}^{(3)}\right) & , \nu \rightarrow \infty\end{cases}
$$

while, the value function under a compulsive strategy is indicated in (17), which yields (B-12).

$$
\Phi_{1}^{(1)}(P)= \begin{cases}U\left(P D_{1}\right)-\frac{U\left(c D_{1}+r I_{1}^{(1)}\right)}{\rho} & , \nu \neq 0 \\ \mathcal{A}^{(2)} U\left(P D_{2}\right)-\frac{U\left(c D_{2}+r I_{1}^{(1)}\right)}{\rho}+B_{0}^{(1)} P^{\beta_{22}} & , \nu \rightarrow \infty\end{cases}
$$

From (B-11) and (B-12) we have: $\nu=0 \Rightarrow \Phi_{1}^{(1)}(P)>\widehat{F}_{1}^{(1)}(P)=0$, while $\nu \rightarrow \infty \Rightarrow \Phi_{1}^{(1)}(P)<$ $\widehat{F}_{1}^{(1)}(P)$. Note that $\nu \nearrow \Rightarrow \widehat{F}_{1}^{(1)}(P) \nearrow$, and, as a result, $\forall P \geq \widehat{p}_{1}^{(3)}, \exists \bar{\nu} \in[0,1]: \widehat{F}_{1}^{(1)}(P) \geq \Phi_{1}^{(1)}(P)$, $\forall \nu \geq \bar{\nu}$. This implies that $\widehat{F}_{1}^{(1)}(P)$ is more responsive to changes in $\nu$ than $\Phi_{1}^{(1)}(P)$. However, $\gamma \searrow \Rightarrow \widehat{F}_{1}^{(1)}(P) \searrow$ and $\Phi_{1}^{(1)}(P) \searrow$. In addition, lower $\gamma$ raises risk aversion, and, in turn, the concavity of the value function under both strategies. This implies that a greater price and $\bar{\nu}$ are required so that $\widehat{F}_{1}^{(1)}(P) \geq \Phi_{1}^{(1)}(P)$. Consequently, the set, $\mathbb{G}_{n}$, of values of $\gamma$ for which the leapfrog/laggard strategy dominates the compulsive strategy increases as the output price increases.

\section{References}

[1] Ang, JB (2014), "Innovation and Financial Liberalisation," Journal of Banking \& Finance, 47: 214-229.

[2] Alvarez, L and R Stenbacka (2004), "Optimal Risk Adoption: A Real Options Approach," Economic Theory, 23: 123-147.

[3] Alvarez, L and R Stenbacka (2001), "Adoption of Uncertain Multi-Stage Technology Projects: A Real Options Approach," Journal of Mathematical Economics, 35: 71-79.

[4] Balcer, Y and S Lippman (1984), "Technological Expectations and Adoption of Improved Technology," Journal of Economic Theory, 34: 292-318.

[5] Bethuyne, G (2002), "The Timing of Technology Adoption by a Cost-Minimizing Firm," Journal of Economics, 76(2): 123-154.

[6] Bos, JWB, JW Kolari, and RCR Lamoen (2013), "Competition and Innovation: Evidence from Financial Services", Journal of Banking \&3 Finance, 37(5): 1590-1601. 
[7] Bowman, E (1982), "Risk Seeking by Troubled Firms", Sloan Management Review, 23(4): 33-42.

[8] Bromlay, P (1991), "Testing a Causal Model of Corporate Risk Taking and Performance," Academy of Management Journal, 34(1): 37-59.

[9] Chronopoulos, M, and A Siddiqui (2015), "When Is It Better to Wait for a New Version? Optimal Replacement of an Emerging Technology Under Uncertainty," Annals of Operations Research 235(1): $177-201$.

[10] Chronopoulos, M, B De Reyck, and AS Siddiqui (2014), "Duopolistic Competition under Risk Aversion and Uncertainty," European Journal of Operational Research, 236(2): 643-656.

[11] Chronopoulos, M, B De Reyck, and AS Siddiqui (2013), "The Value of Capacity Sizing Under Risk Aversion and Operational Flexibility," IEEE Transactions on Engineering Management, 60(2): 272288 .

[12] Chronopoulos, M, B De Reyck, and AS Siddiqui (2011), "Optimal Investment under Operational Flexibility, Risk Aversion, and Uncertainty," European Journal of Operational Research, 213(1): 221-237.

[13] Dangl, T (1999), "Investment and Capacity Choice under Uncertain Demand," European Journal of Operational Research, 117: 415-428.

[14] Décamps, JP, T Mariotti, and S Villeneuve (2006), "Irreversible Investment in Alternative Projects," Economic Theory, 28: 425-448.

[15] Dixit, AK and RS Pindyck (1994), Investment under Uncertainty, Princeton University Press, Princeton, NJ, USA.

[16] Dixit, AK (1993), "Choosing Among Alternative Discrete Investment Projects under Uncertainty," Economics Letters, 41: 265-268.

[17] Doraszelski, U (2004), "Innovations, Improvements, and the Optimal Adoption of New Technologies," Journal of Economic Dynamics \& Control, 28(7): 1461-1480.

[18] Doraszelski, U (2001), "The Net Present Value Method versus the Option Value of Waiting: A Note on Farzin, Huisman and Kort (1998)," Journal of Economic Dynamics E Control, 25(8): 1109-1115.

[19] Farzin, Y, KJM Huisman, and PM Kort, (1998) "Optimal Timing of Technology Adoption," Journal of Economic Dynamics \& Control, 22: 779-799.

[20] Fleten, SE, KM Maribu, and I Wangensteen (2007), "Optimal Investment Strategies in Decentralised Renewable Power Generation under Uncertainty," Energy, 32(5): 803-815.

[21] Goto, M, K Nishide, and R Takashima (2013), "Irreversible Investment under Competition with Markov Switching," discussion paper No. 861, Kyoto University, Kyoto, Japan. 
[22] Grenadier, SR and AM Weiss (1997), "Investment in Technological Innovations: An Option Pricing Approach," Journal of Financial Economics, 44: 397-416.

[23] Hagspiel, V, P Jesus, PM Kort, and C Nunes (2013), "Technological Change: A Burden or a Chance," working paper, Norwegian University of Science and Technology, Trondheim, Norway.

[24] Henderson, V (2007), "Valuing the Option to Invest in an Incomplete Market," Mathematics and Financial Economics, 1: 103-128.

[25] Henderson, V and D Hobson (2002), "Real Options with Constant Relative Risk/Aversion," Journal of Economic Dynamics \& Control, 27: 239-355.

[26] Hoppe, HC (2002), "The Timing of New Technology Adoption: Theoretical Models and Empirical Evidence," The Manchester School, 70(1): 1463-6786.

[27] Hugonnier, J and E Morellec (2013), "Real Options and Risk Aversion," Real Options, Ambiguity, Risk and Insurance, ISBN-13: 978-1614992370.

[28] Huisman, K and PM Kort (2004), "Strategic Technology Adoption Taking into Account Future Technological Improvements: A Real Options Approach”, European Journal of Operational Research, 159: $705-728$

[29] Huisman, K and PM Kort (2003), "Strategic Investment in Technological Innovations", European Journal of Operational Research, 144: 209-223.

[30] Karatzas, I and S Shreve (1999), Methods of Mathematical Finance, Springer Verlag, New York, NY, USA.

[31] Koussis, N, SH Martzoukos, and L Trigeorgis (2013), "Multi-Stage Product Development with Exploration, Value-Enhancing, Pre-Emptive and Innovation Options", Journal of Banking E Finance, 37: $174-190$.

[32] McDonald, RL and DS Siegel (1986), "The Value of Waiting to Invest," The Quarterly Journal of Economics, 101(4): 707-728.

[33] McDonald, RL and DS Siegel (1985), "Investment and Valuation of Firms When There is an Option to Shut Down," International Economic Review, 26(2): 331-349.

[34] Merton, RC, (1969), "Lifetime Portfolio Selection under Uncertainty: The Continuous Time Case," Review of Economics and Statistics, 51: 247-257.

[35] Nawrocki, D (2002), "The Case for the Relevancy of Downside Risk Measures," The Handbook of Risk, $79-96$. 
[36] Parente, SL (1994), "Technology Adoption, Learning-by-Doing and Economic Growth," Journal of Economic Theory, 63: 346-369.

[37] Pratt, JW (1964), "Risk Aversion in the Small and in the Large", Econometrica, 32(1-2): 122-136.

[38] Siddiqui, A and SE Fleten (2010), "How to Proceed with Competing Alternative Energy Technologies: A Real Options Analysis," Energy Economics, 23: 817-830.

[39] The Economist (2012a), "The last Kodak moment?," 14 January.

[40] The Economist (2012b), "Gone in a Flash," 6 May. 